\title{
Decentralized, Deterministic Robot Swarm Control using Blob Methods for PDEs
}

July 10, 2020

\section{Matt Haberland $^{1 *}$, Katy Craig $^{2}$, Karthik Elamvazhuthi $^{3}$, Olga Turanova $^{4}$}

1. BioResource and Agricultural Engineering Department, Cal Poly

2. Department of Mathematics, University of California, Santa Barbara

3. Department of Mathematics, University of California, Los Angeles

4. Department of Mathematics, Michigan State University

* Corresponding Author, mhaberla@calpoly.edu

\section{Introduction}

As the technology to create swarms of inexpensive robots matures, the need for swarm control algorithms arises. One control problem of particular interest is that of coverage: how should individual robots behave for the swarm to achieve a prescribed density in each portion of a domain? There is at least one obvious solution: a central server determines a desired position of each robot, and each robot moves to the assigned location. But when the number of robots is very large or when the communication capability of robots is limited, a decentralized controller would be preferred. Here, we investigate an approach based on a numerical solution method for partial differential equations.

We begin by expressing the density of robots mathematically as the equilibrium solution $\bar{\rho}(x)$ of a diffusive PDE

$$
\frac{\partial \rho}{\partial t}-\Delta\left(\frac{\rho}{\bar{\rho}}\right)^{m}=0
$$

over domain $\Omega \subset \mathbb{R}^{d}$ with no-flux boundary condition and $m \geq 1$. For example, if our domain is the unit square in the plane and we want our robots to be distributed uniformly, $\bar{\rho}(x)=1, x \in \Omega$.

In blob methods for PDEs [1], the initial condition of such a PDE is expressed as a sum of discrete Dirac masses, and the PDE reduces to a system of ordinary differential equations that govern the movement of the Dirac masses over time. Here, we use the ordinary differential equations governing the Dirac mass movements to prescribe the velocities of simulated robots, and this control law guides the swarm to achieve the desired distribution $\bar{\rho}(x)$. After presenting an implementation of the control law and swarm simulation, we describe the computational experiments in progress and discuss improvements to the computational efficiency. 


\subsection{The Mollifier}

In many swarm coverage applications, a robot's "mass", or influence, is not considered to be concentrated at a single point, but is instead considered to be distributed axisymmetrically according to some mollifier function $\phi(x)$, such as the Gaussian $\phi(x)=\frac{1}{\sqrt{2 \pi}} e^{-x^{2} / 2}$.

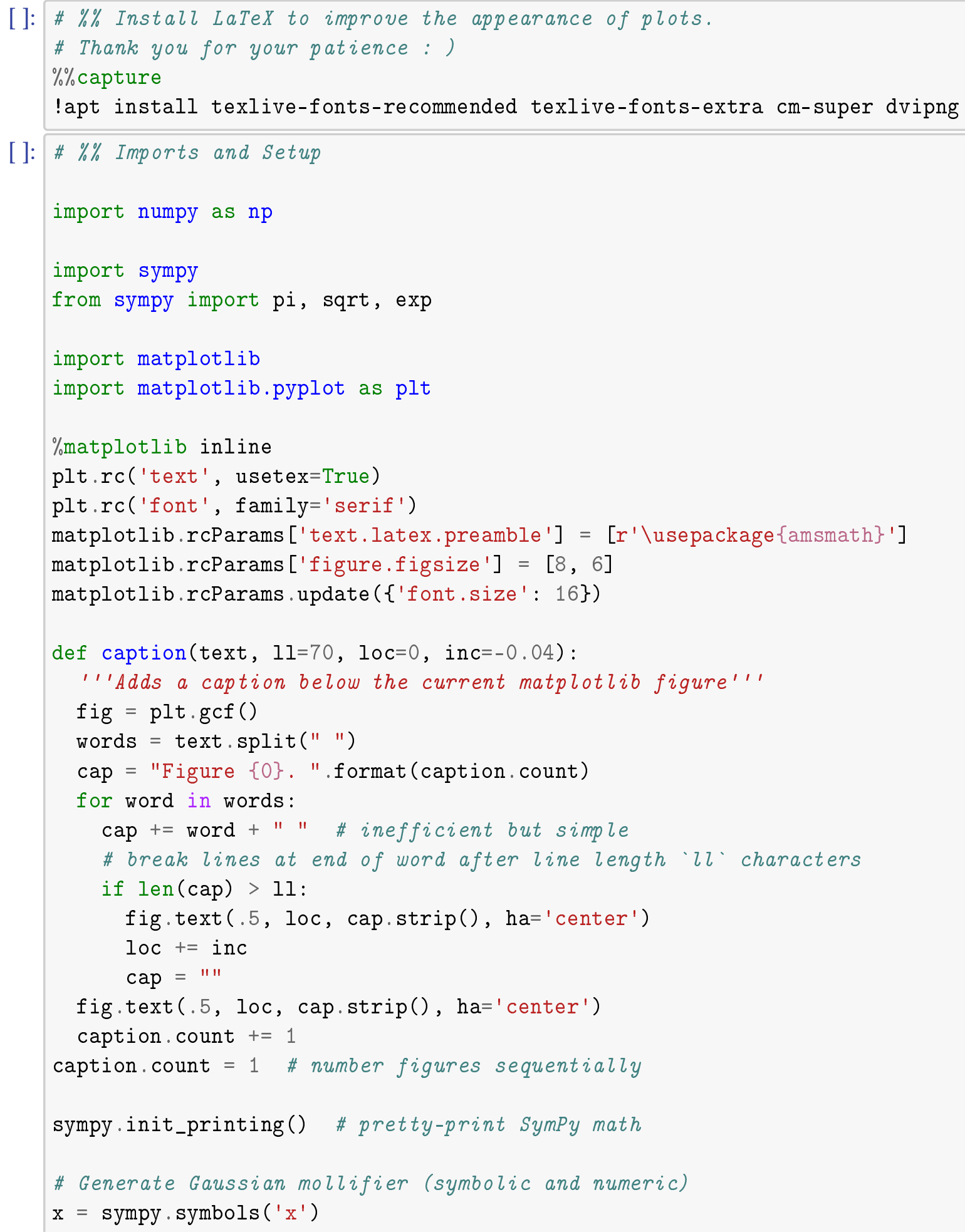



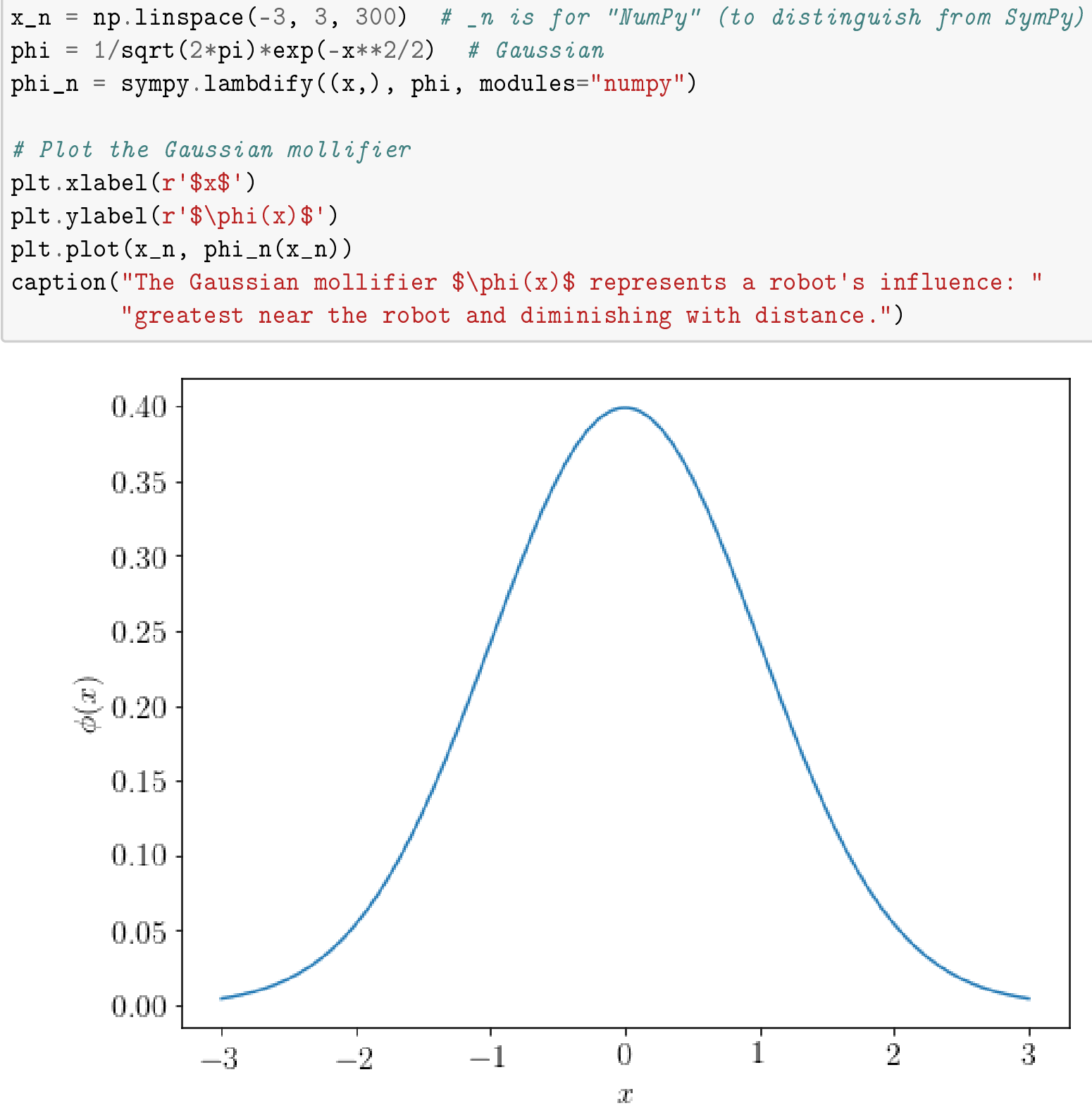

Figure 1. The Gaussian mollifier $\phi(x)$ represents a robot's influence: greatest near the robot and diminishing with distance.

\subsection{The Robot Blob Function}

If the $i^{\text {th }}$ robot in a swarm is located at position $x_{i}$ and a robot's influence has a radius parameter $\epsilon$ that characterizes how quickly the influences fades as the distance from the robot increases, then associated with each robot is a robot blob function $\phi_{\epsilon}\left(x, x_{i}\right)=\frac{1}{\epsilon^{d}} \phi\left(\frac{x-x_{i}}{\epsilon}\right)$.

[]: \# Generate robot blob function (symbolic and numeric)

xi, eps = sympy.symbols ('x_i, \\epsilon') 

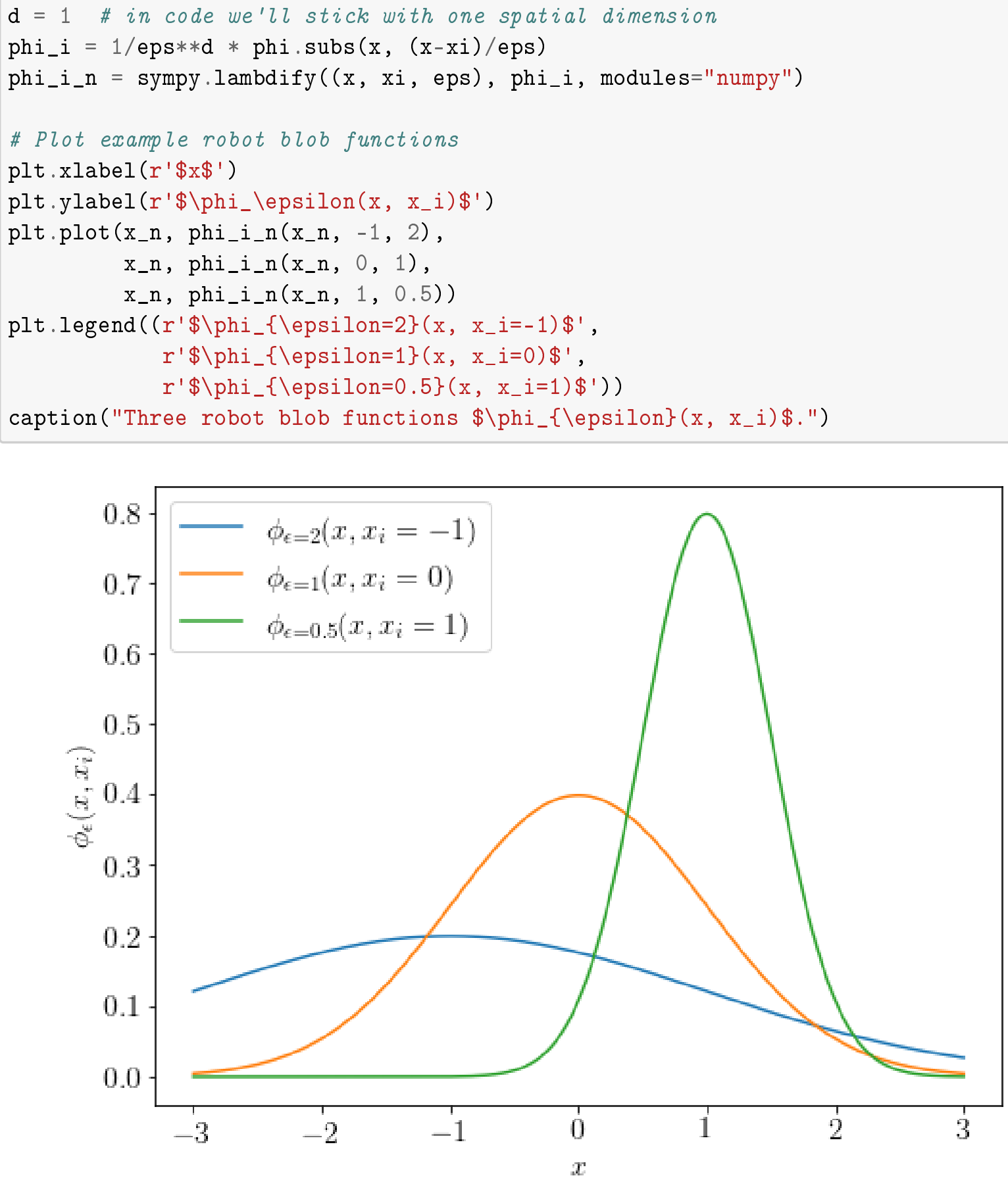

Figure 2. Three robot blob functions $\phi_{\epsilon}\left(x, x_{i}\right)$.

\subsection{The Swarm Blob Function}

We define the swarm blob function as a weighted sum of the influences of all the robots, 


$$
\rho_{\epsilon}(x)=\sum_{i} \phi_{\epsilon}\left(x, x_{i}\right) m_{i}
$$

where $m_{i}$ is the $i^{\text {th }}$ robot's mass, which quantifies the strength of the robot's influence. The swarm blob function allows us to unambiguously quantify at every point in a domain the "density" of a finite number of discrete robots.

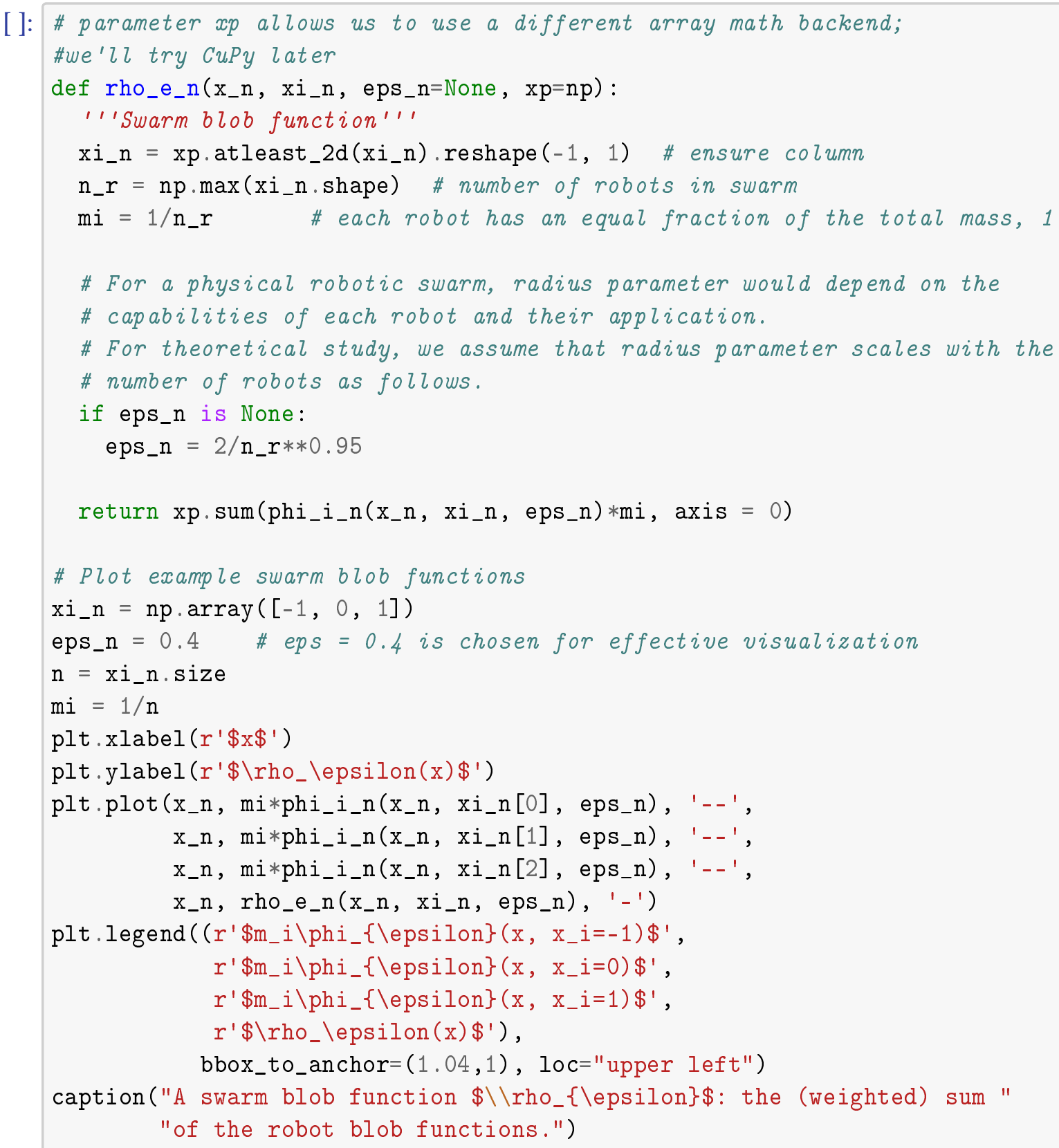




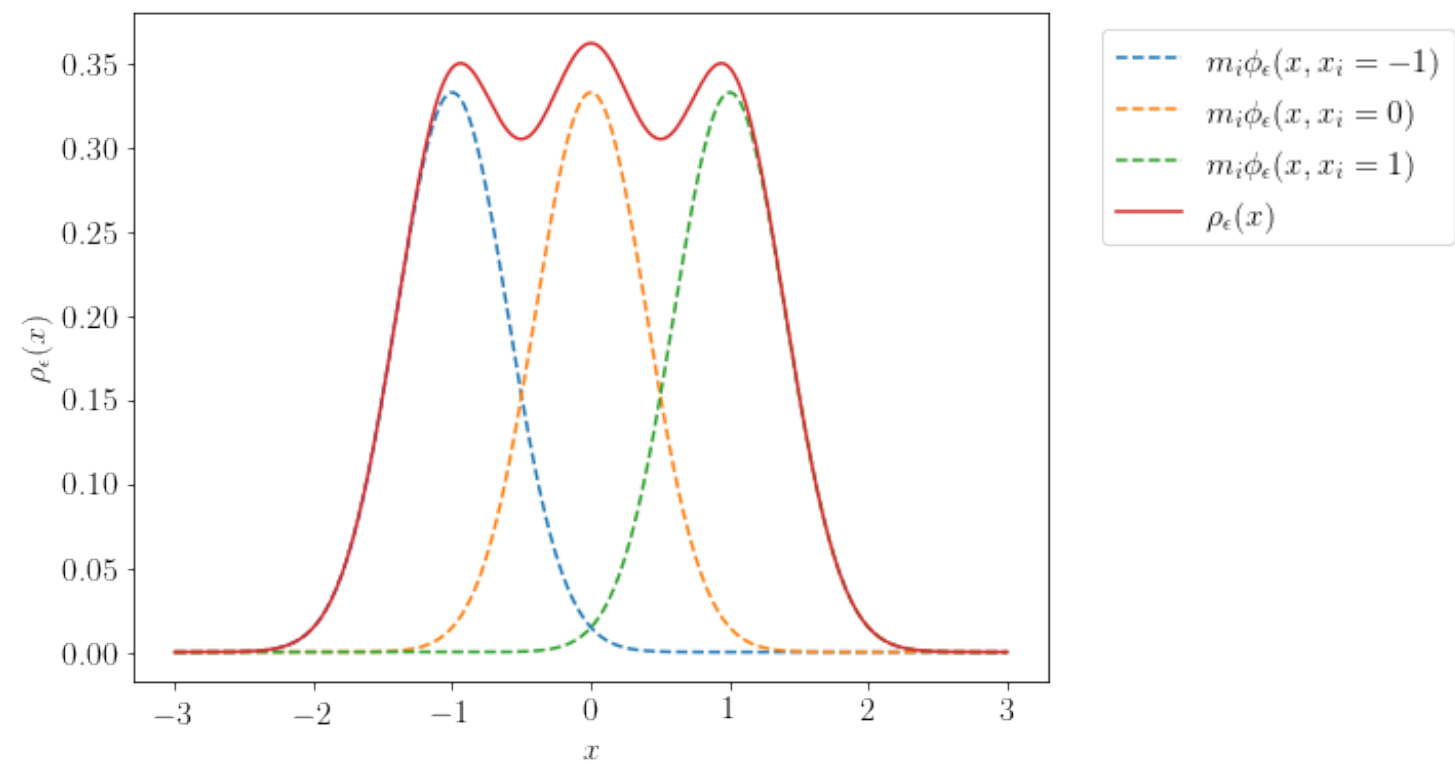

Figure 3. A swarm blob function $\rho_{\epsilon}$ : the (weighted) sum of the robot blob functions.

\subsection{The Control Law}

In terms of these functions, the control law derived from (1) with $m=2$ can be written

$$
v_{i}=-a\left(x_{i}\right)\left(\rho_{\epsilon}\left(x_{i}\right) \nabla a\left(x_{i}\right)+a\left(x_{i}\right) \nabla \rho_{\epsilon}\left(x_{i}\right)+\sum_{j} m_{j} \nabla \phi_{\epsilon}\left(x_{i}, x_{j}\right) a\left(x_{j}\right)\right),
$$

where $a(x)=\frac{1}{\bar{\rho}(x)}$ and $v_{i}$ is the instantaneous velocity of the $i^{\text {th }}$ robot.

\section{Simulation}

\subsection{Initial Distribution}

Suppose that $n_{r}=10$ robots in a one-dimensional world are initially clustered about the origin.

[]: \# Generate initial configuration of 10 robots and plot swarm blob function $\mathrm{np} \cdot \mathrm{random} \cdot \operatorname{seed}(4)$

n_r $=10$

$\mathrm{xi} 0=\mathrm{np} \cdot \mathrm{random} \cdot \operatorname{rand}\left(\mathrm{n}_{-} \mathrm{r}\right)-0.5$

plt.plot(xi_0, np.zeros_like(xi_0), '.', $x_{-} n$, rho_e_n $\left.\left(x_{-} n, x_{-} 0\right),{ }^{\prime}{ }^{\prime}\right)$

plt.xlabel $\left(r^{\prime} \$ \mathrm{x} \${ }^{\prime}\right)$

plt.ylabel ( $r^{\prime} \$ \backslash r h o_{-} \backslash$ epsilon ( $x$ )\$')

plt.legend(("Initial Robot Position", "Swarm Blob Function"))

caption("Example robot positions and corresponding swarm blob function.") 


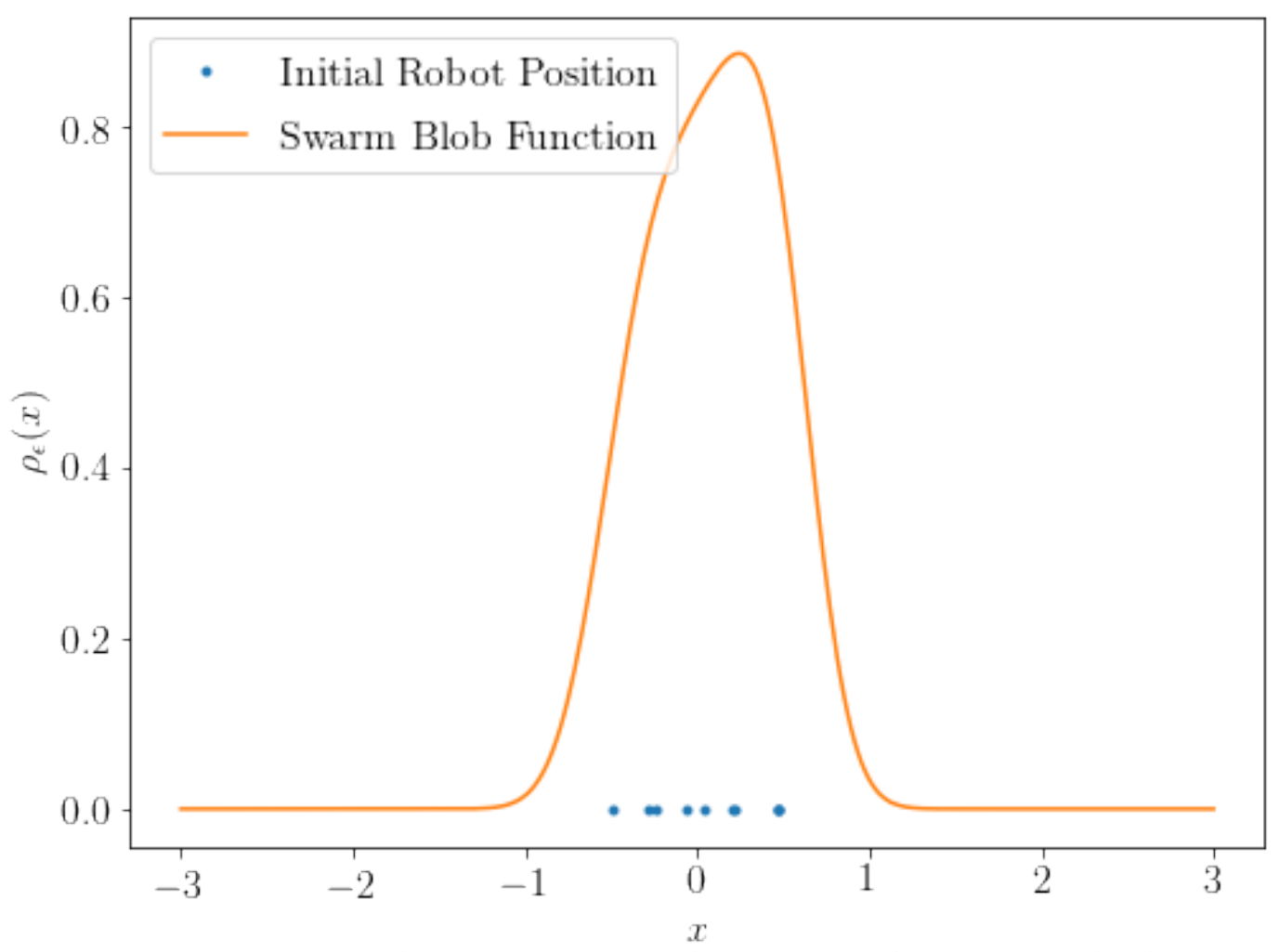

Figure 4. Example robot positions and corresponding swarm blob function.

\subsection{Target Distribution}

We wish for the robots to distribute themselves according to some target distribution $\bar{\rho}(x)^{\prime}$ within the domain $\Omega=[-2,2]$ according to the control law given by (3). To do this, we express the condition that the robots be distributed uniformly by choosing the target distribution $\bar{\rho}^{\prime}(x)$ to be a constant, e.g. $\bar{\rho}^{\prime}(x)=\frac{1}{4}$. We can enforce the no-flux boundary condition by multiplying our desired distribution by a boxcar function $B_{\Omega}(x)$, which has value one within the domain and zero outside the domain. The equilibrium solution to (1) is therefore

$$
\bar{\rho}(x)=\bar{\rho}^{\prime}(x) B_{\Omega}(x) .
$$

In our one dimensional case, $B_{\Omega}(x)=H(x+2)-H(x-2)$, where $H(x)$ is the Heaviside step function.

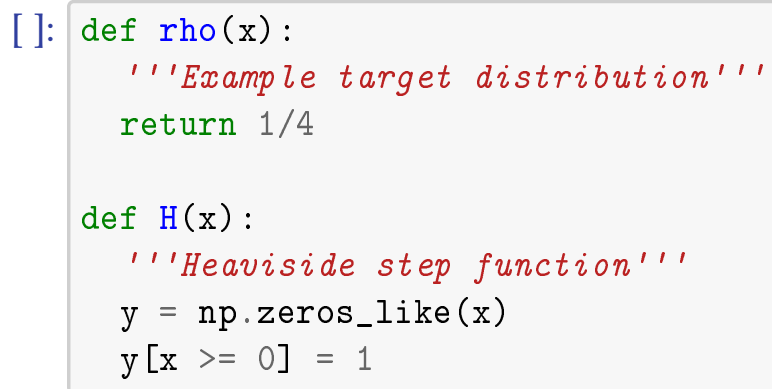



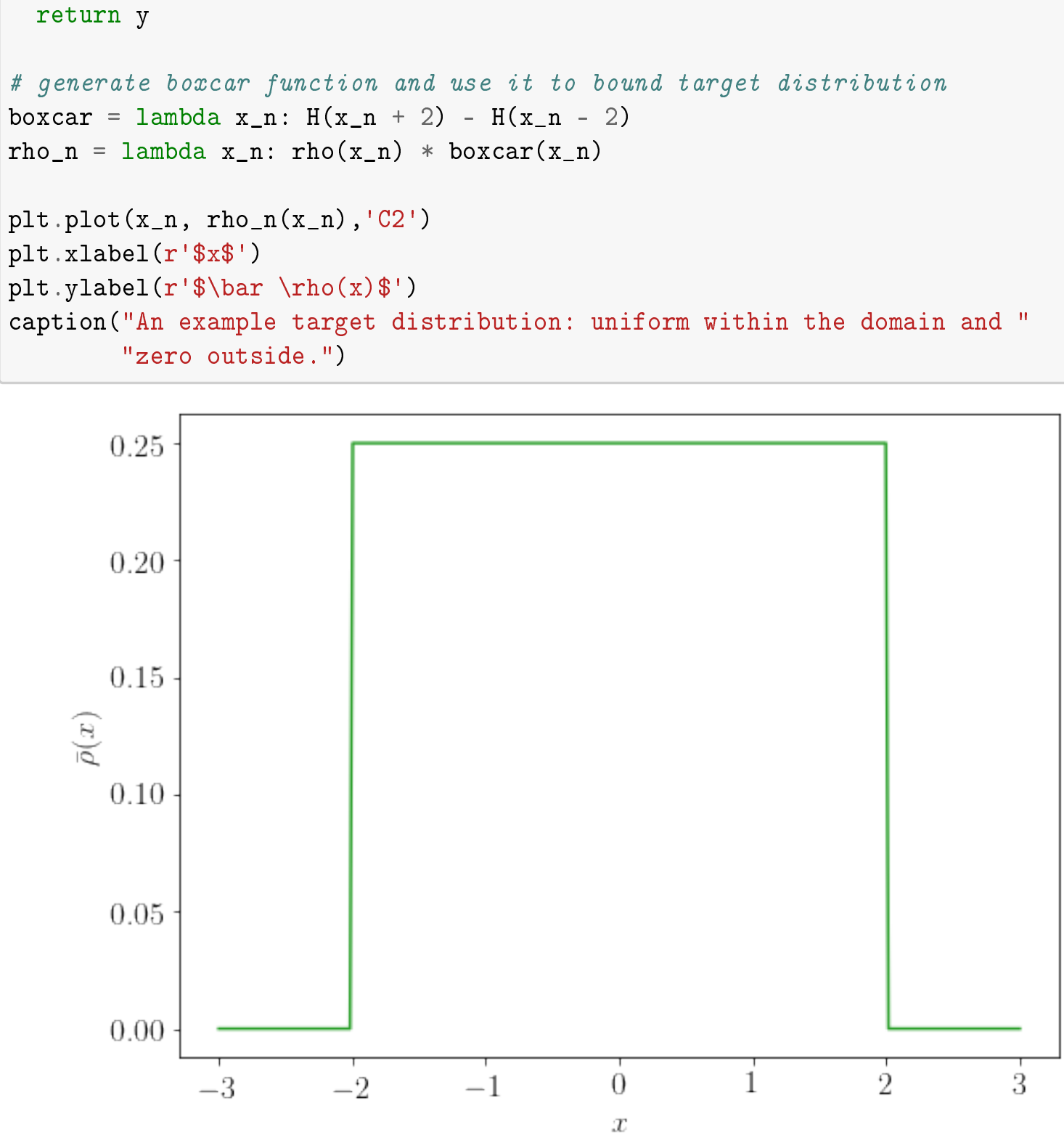

Figure 5. An example target distribution: uniform within the domain and zero outside.

Note that although we have chosen $\bar{\rho}^{\prime}(x)=\frac{1}{4}$ to be normalized such that $\int_{\Omega} \bar{\rho}^{\prime}(x)=1$, this is not strictly required. A target distribution of any constant value simply means that the target density of robots is the same at every point within the domain. The density of the swarm is specified in relative terms (e.g. $\bar{\rho}\left(x_{1}\right)=2$ and $\bar{\rho}\left(x_{2}\right)=4$ means that the swarm's influence is twice as dense at $x_{2}$ than at $x_{1}$ ) rather than as an absolute value (e.g. number of robots per meter).

For simulation, we need to make two adjustments.

1. Our control law (3) involves the gradient $\nabla \rho_{\epsilon}(x)$, so we replace the discontinuous Heaviside step function with a smoothed approximation: the logistic function $\tilde{H}=\frac{1}{2}+\frac{1}{2} \tanh (k x)$, where $k$ is some large constant. 
2. Our control law (3) involves a term $a(x)=\frac{1}{\bar{\rho}(x)}$, so to avoid division by zero in simulation, we add a small positive constant $\delta$.

Thus, in place of the boxcar function above, we use an approximation that is smooth and positive everywhere:

$$
\tilde{B}_{\Omega}(x)=\tilde{H}(x+2)-\tilde{H}(x-2)+\delta .
$$

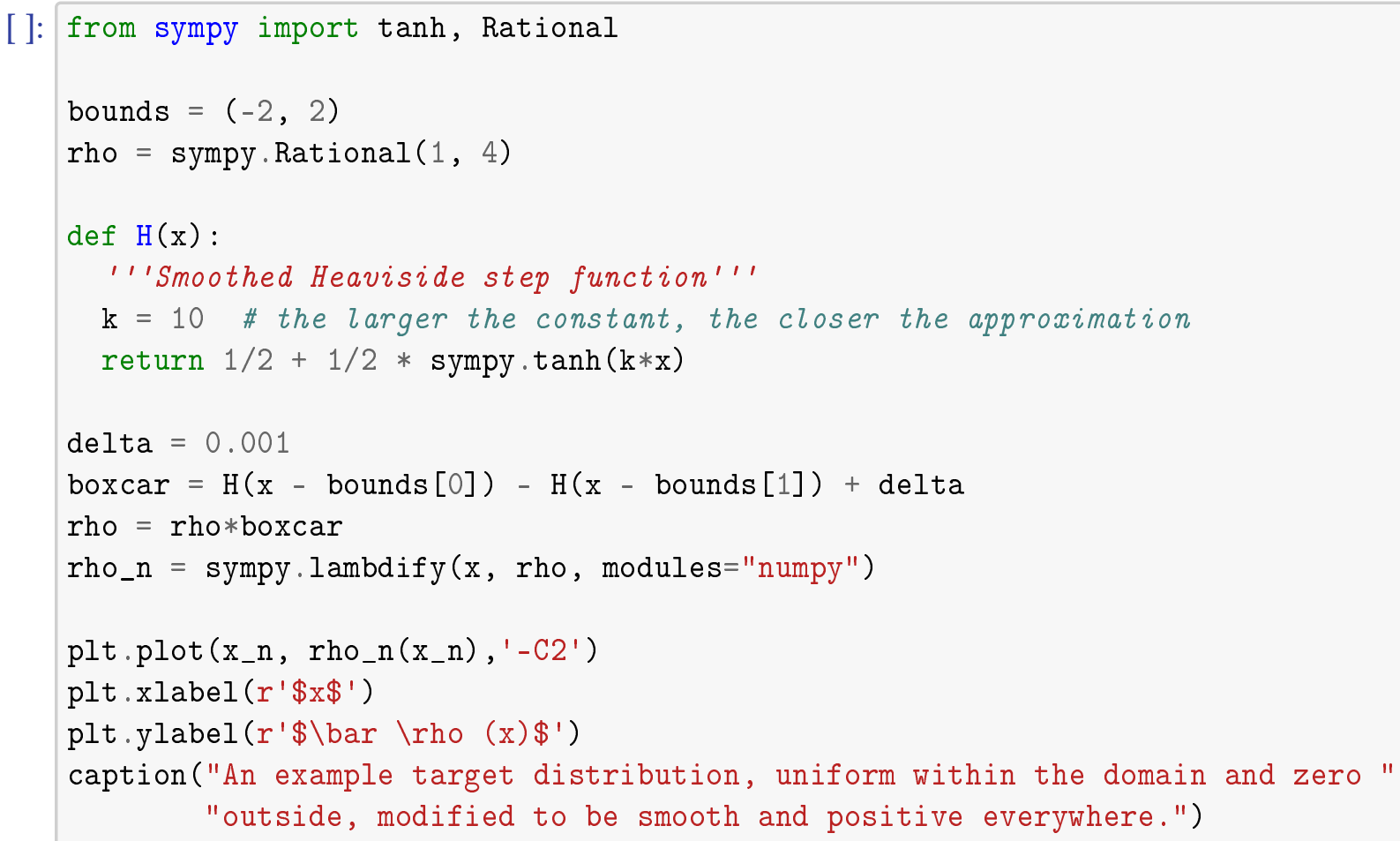




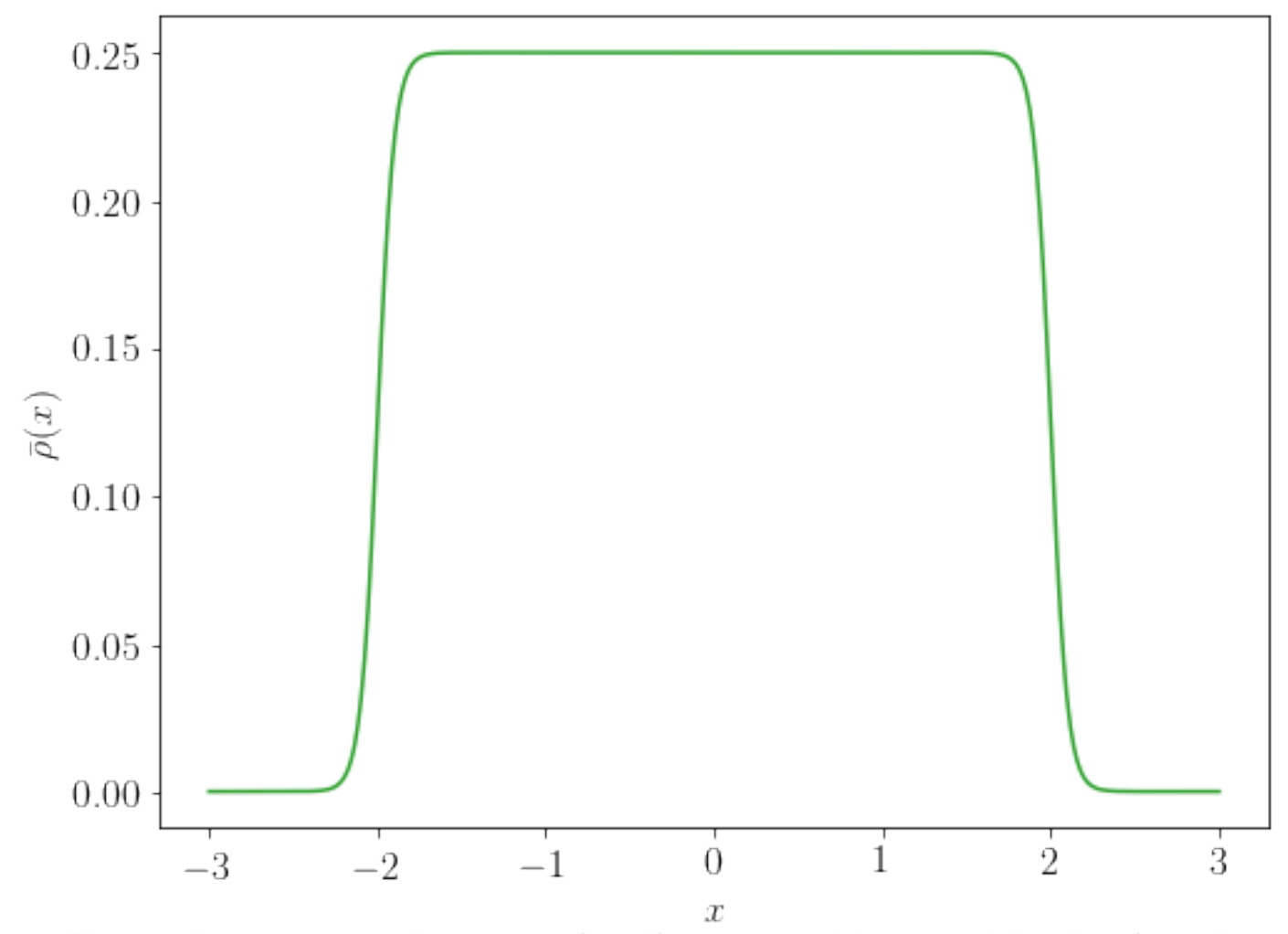

Figure 6. An example target distribution, uniform within the domain and zero outside, modified to be smooth and positive everywhere.

\subsection{Control Law Implementation}

We define $a(x)=\frac{1}{\bar{\rho}(x)}$, take derivatives symbolically, and create numerical functions.

\section{[]: from IPython.display import display}

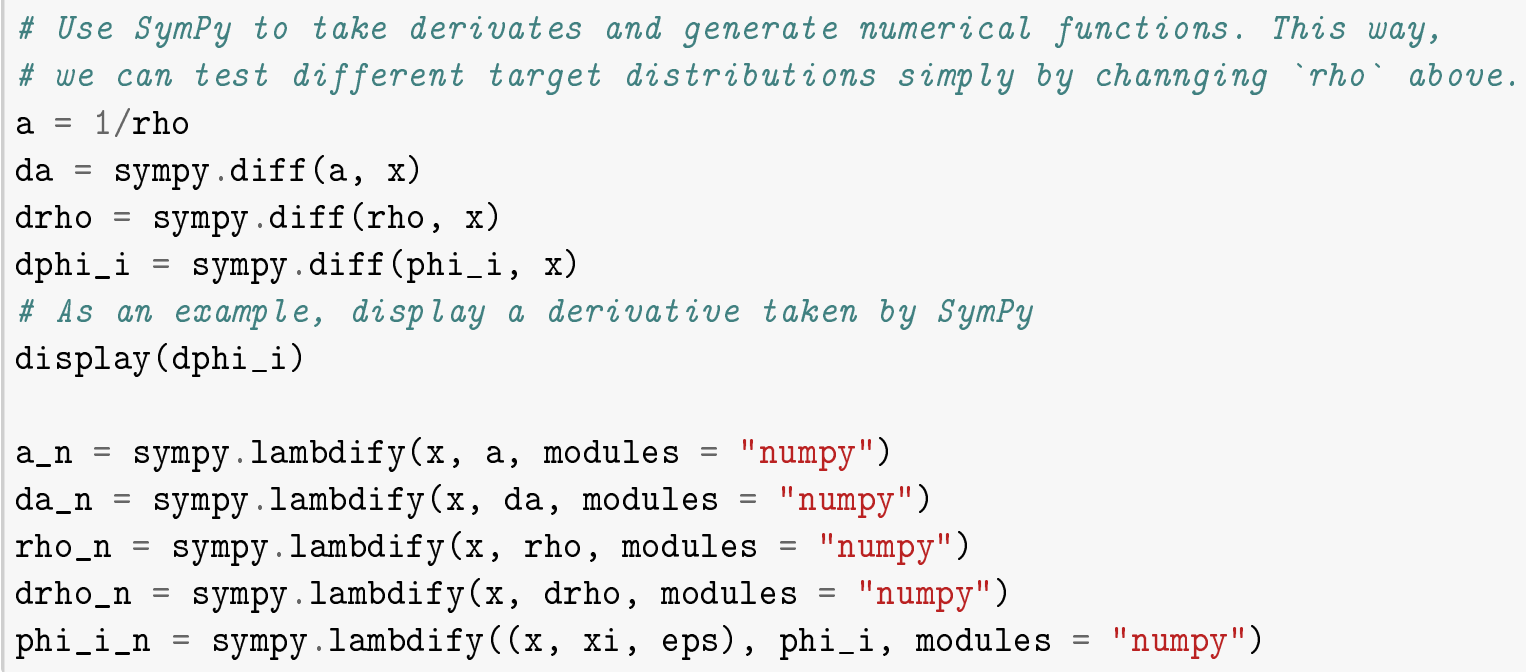




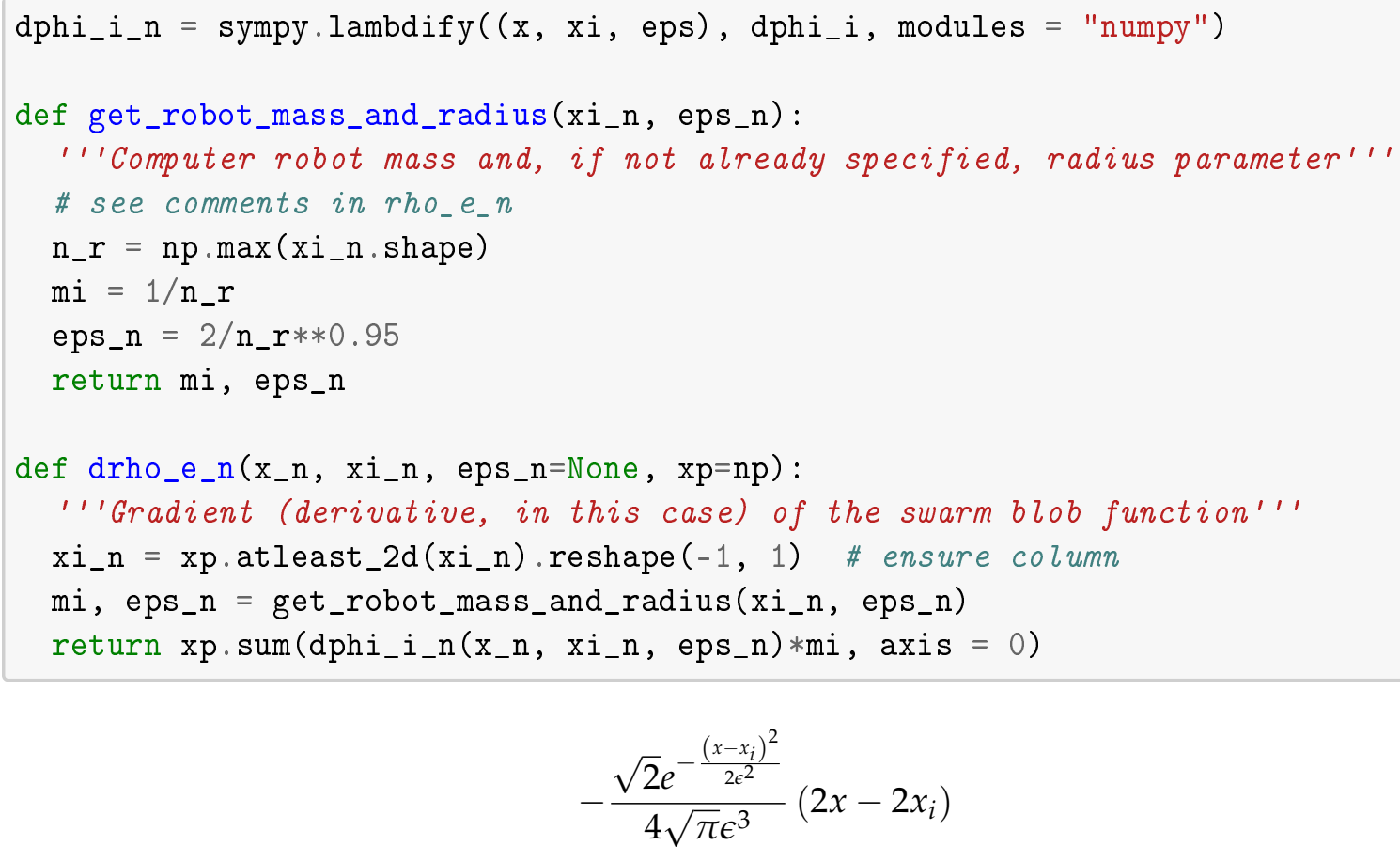

$$
-\frac{\sqrt{2} e^{-\frac{\left(x-x_{i}\right)^{2}}{2 \epsilon^{2}}}}{4 \sqrt{\pi} \epsilon^{3}}\left(2 x-2 x_{i}\right)
$$

Using these, we can define a function that calculates the velocity of each robot given the position of each robot.

[]: def $v\left(x i \_n\right.$, eps_n=None, $\left.x p=n p\right)$ :

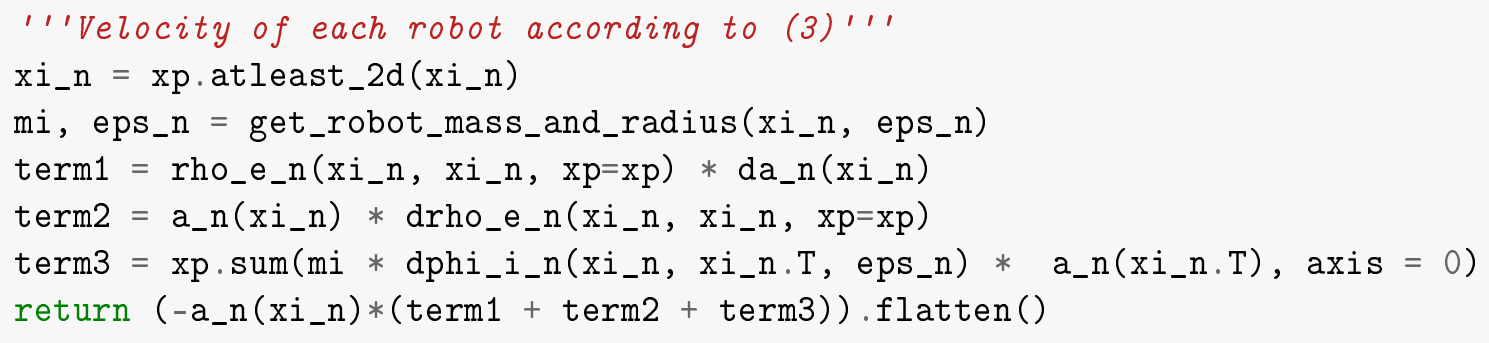

\subsection{ODE Solution}

Finally, we can integrate the velocities of the robots over time to get their final positions.

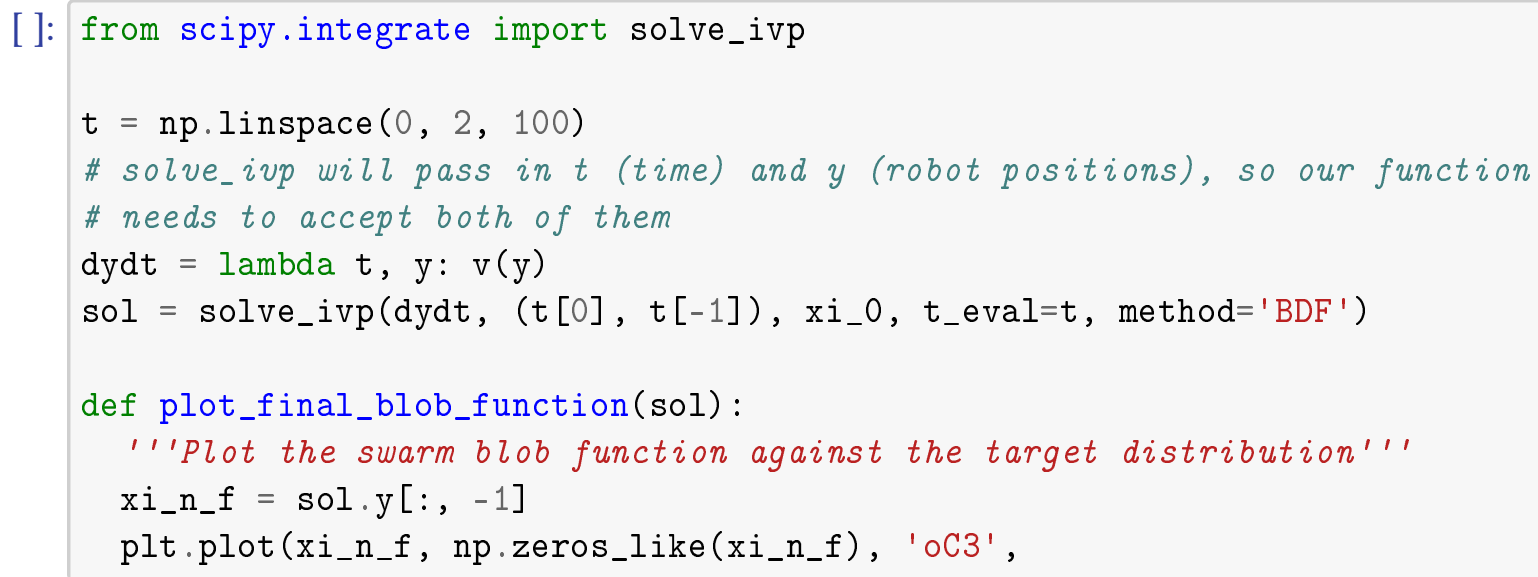



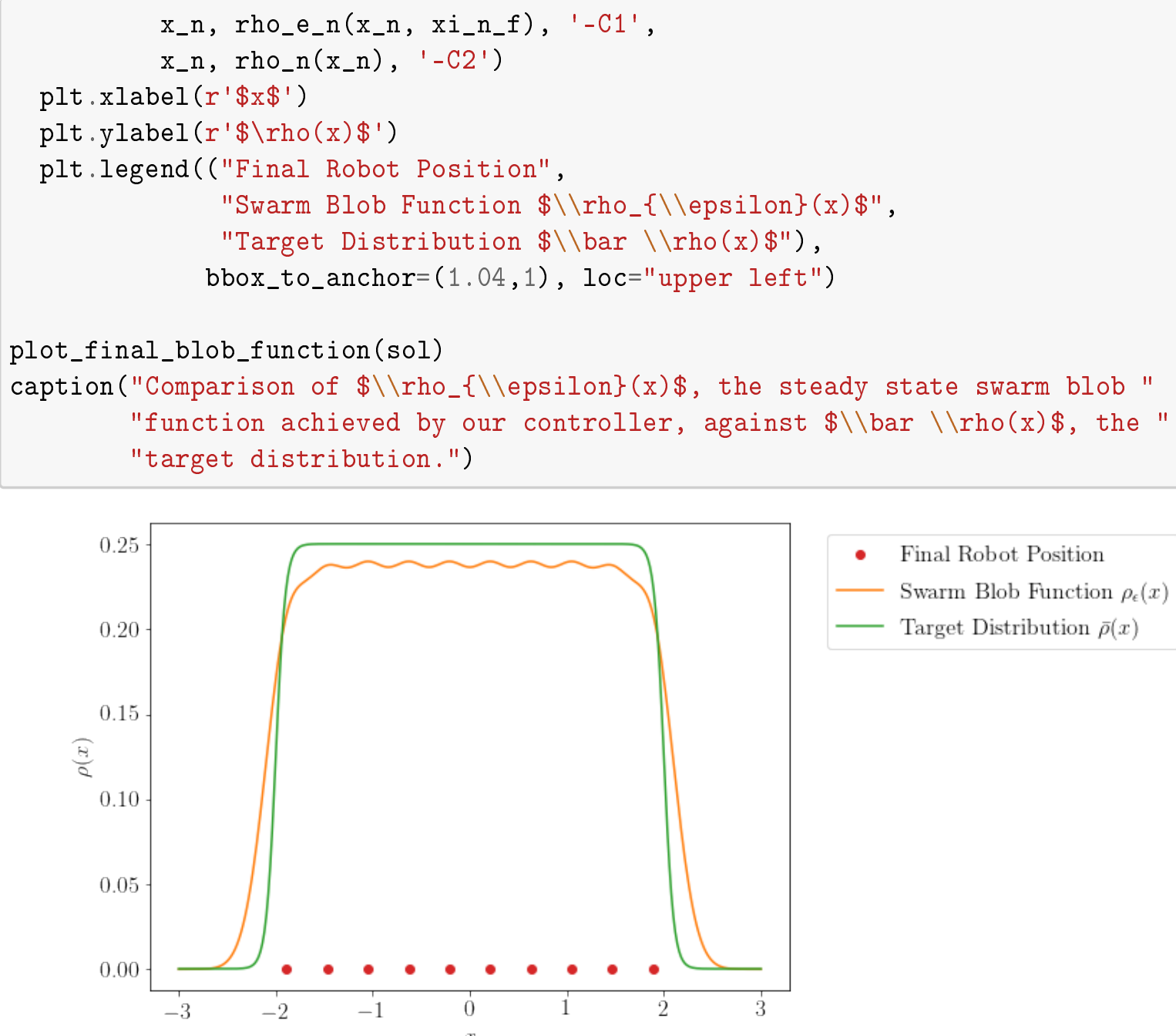

- Final Robot Position

- Swarm Blob Function $\rho_{\epsilon}(x)$

Target Distribution $\bar{\rho}(x)$

Figure 7. Comparison of $\rho_{\epsilon}(x)$, the steady state swarm blob function achieved by our controller, against $\bar{\rho}(x)$, the target distribution.

Note that robot masses have been chosen such that the total swarm mass, the integral under the swarm blob function, is equal to that under the target distribution. However, because the number of robots is small and their influence radius parameter $\epsilon$ is large, the robot blob functions are rather diffuse and some of this mass 'leaks' beyond the edges of the domain. Consequently, the height of the swarm blob function does not reach the targeted value of 0.25 within the domain. As the swarm size gets larger and $\epsilon$ gets smaller, this error tends to be reduced.

Note also that the robots rapidly approach their final positions and then settle into a steady state.

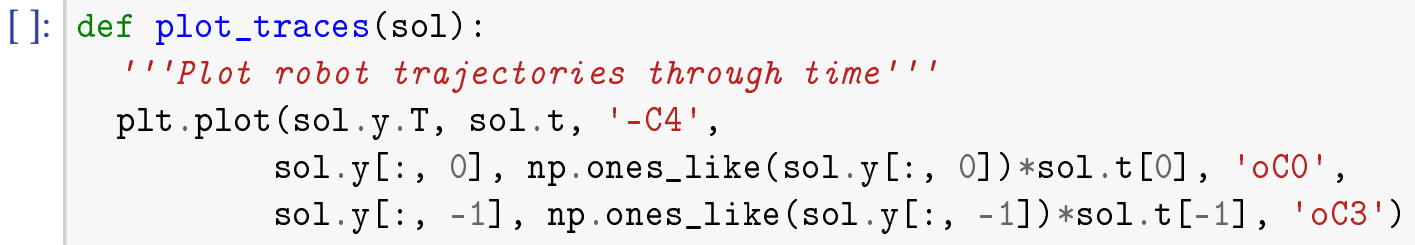




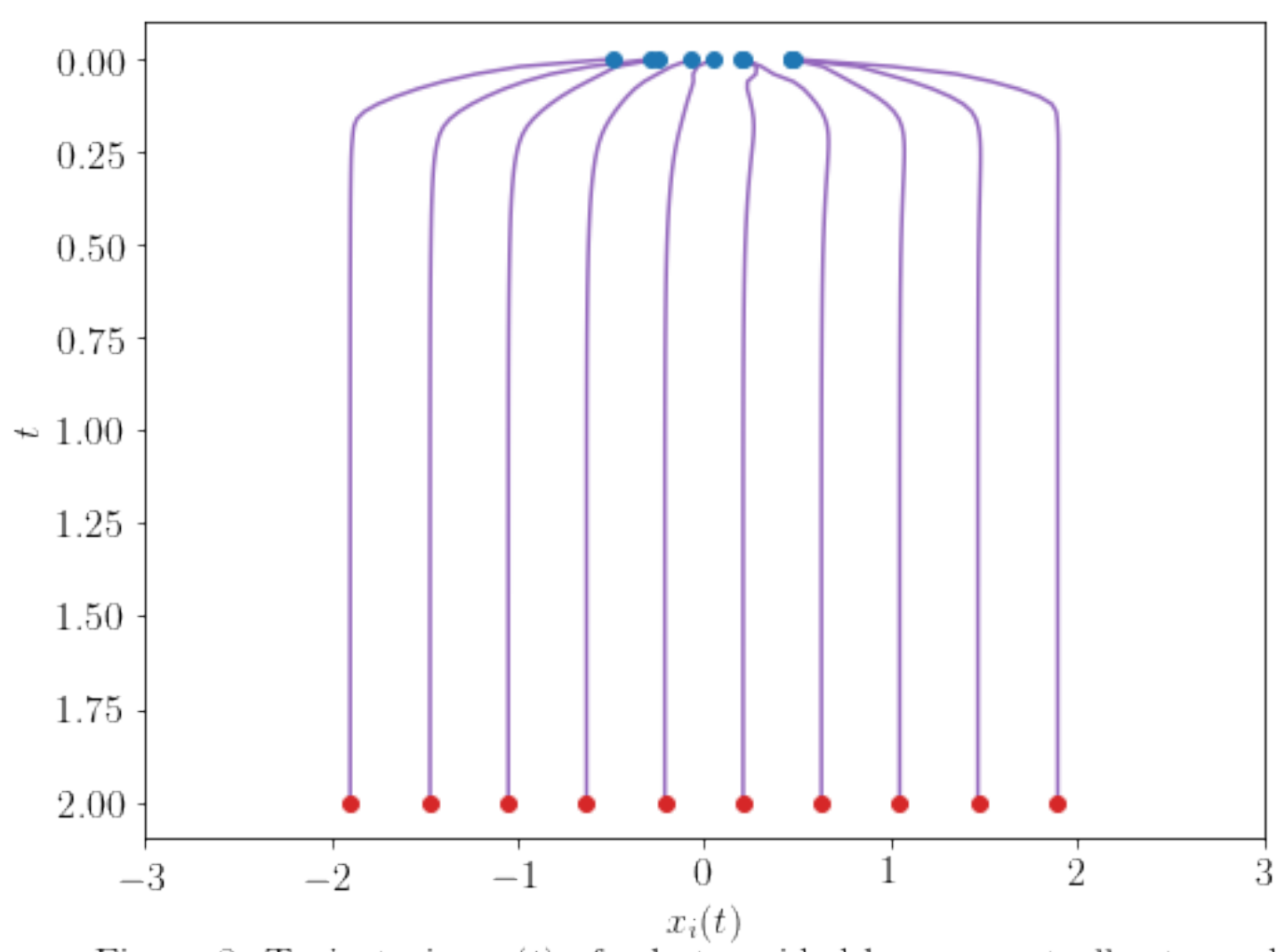

Figure 8. Trajectories $x_{i}(t)$ of robots guided by our controller toward a uniform distribution.

\section{Experiments}

There is a family of control laws similar to (3) that can be developed based on (1) using, for example, different values of $m$ and different mollifier functions $\phi(x)$. Using computational experiments, we wish to compare these different controllers in terms of their speed and accuracy, and for each controller we seek to understand the effects of swarm parameters, such as the number of robots $n$ and the size of each robots' influence $\epsilon$, on these metrics of swarm performance. 


\subsection{Quantifying Accuracy}

One way of quantifying the accuracy of the controller is by comparing the swarm blob function $\rho_{\epsilon}$ against the target distribution $\bar{\rho}$ using the error metric from [2]:

$$
e_{1}(t)=\int_{\Omega}\left|\rho_{\epsilon}(x, t)-\bar{\rho}(x, t)\right| d x
$$

[]: from scipy.integrate import quad

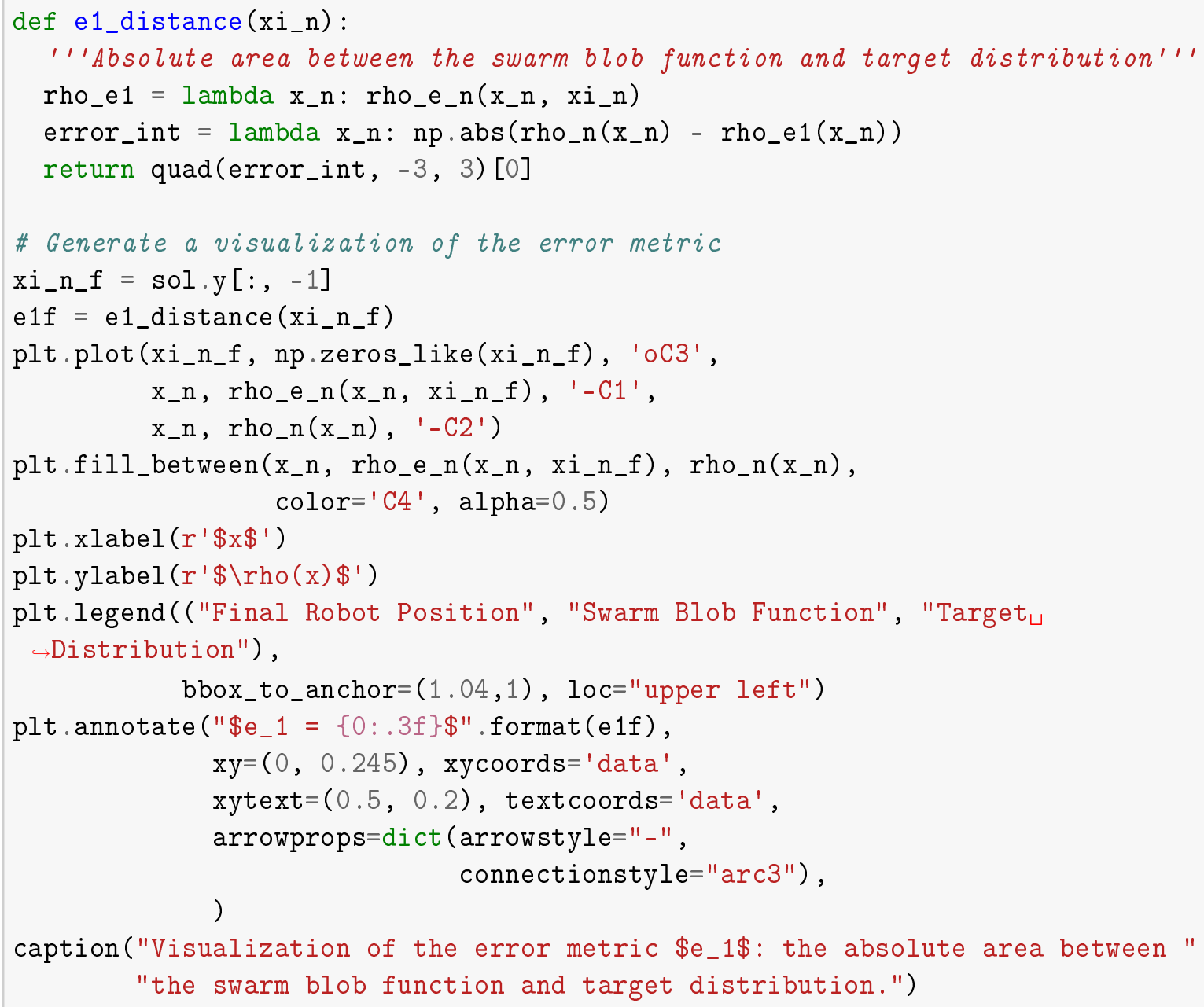




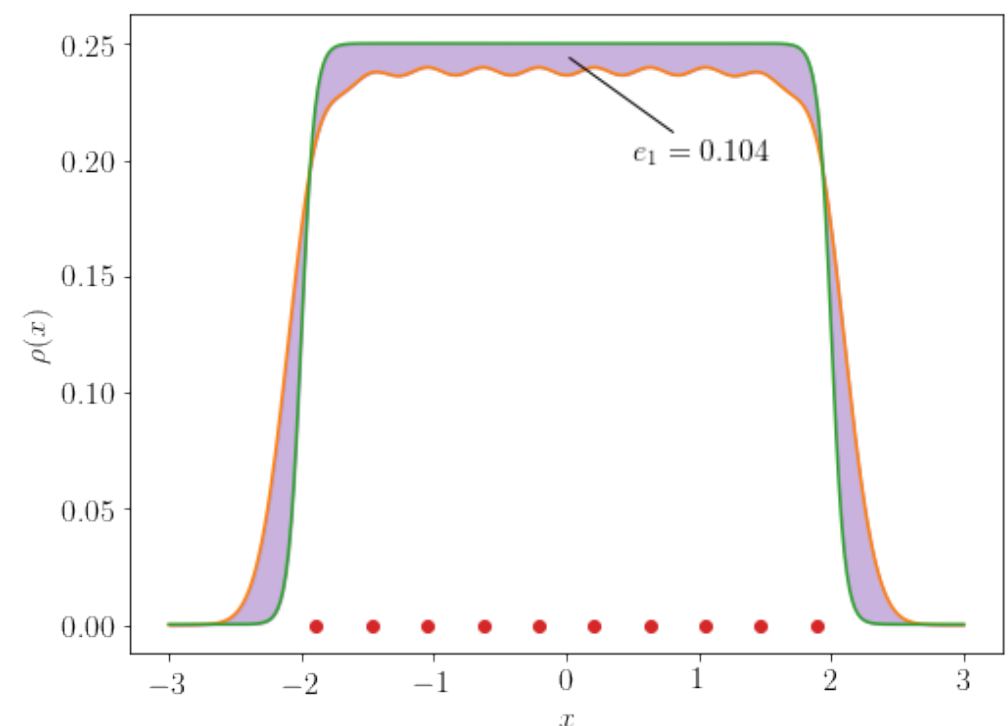

- Final Robot Position

Swarm Blob Function

Target Distribution

Figure 9. Visualization of the error metric $e_{1}$ : the absolute area between the swarm blob function and target distribution.

Alternatively, we can use the Wasserstein ("Earth-Movers") distance, which we denote $e_{2}$, to compare the discrete robot positions against the desired density distribution.

Below, we plot the value of these two metrics as the swarm configuration evolves over time according to the control law.

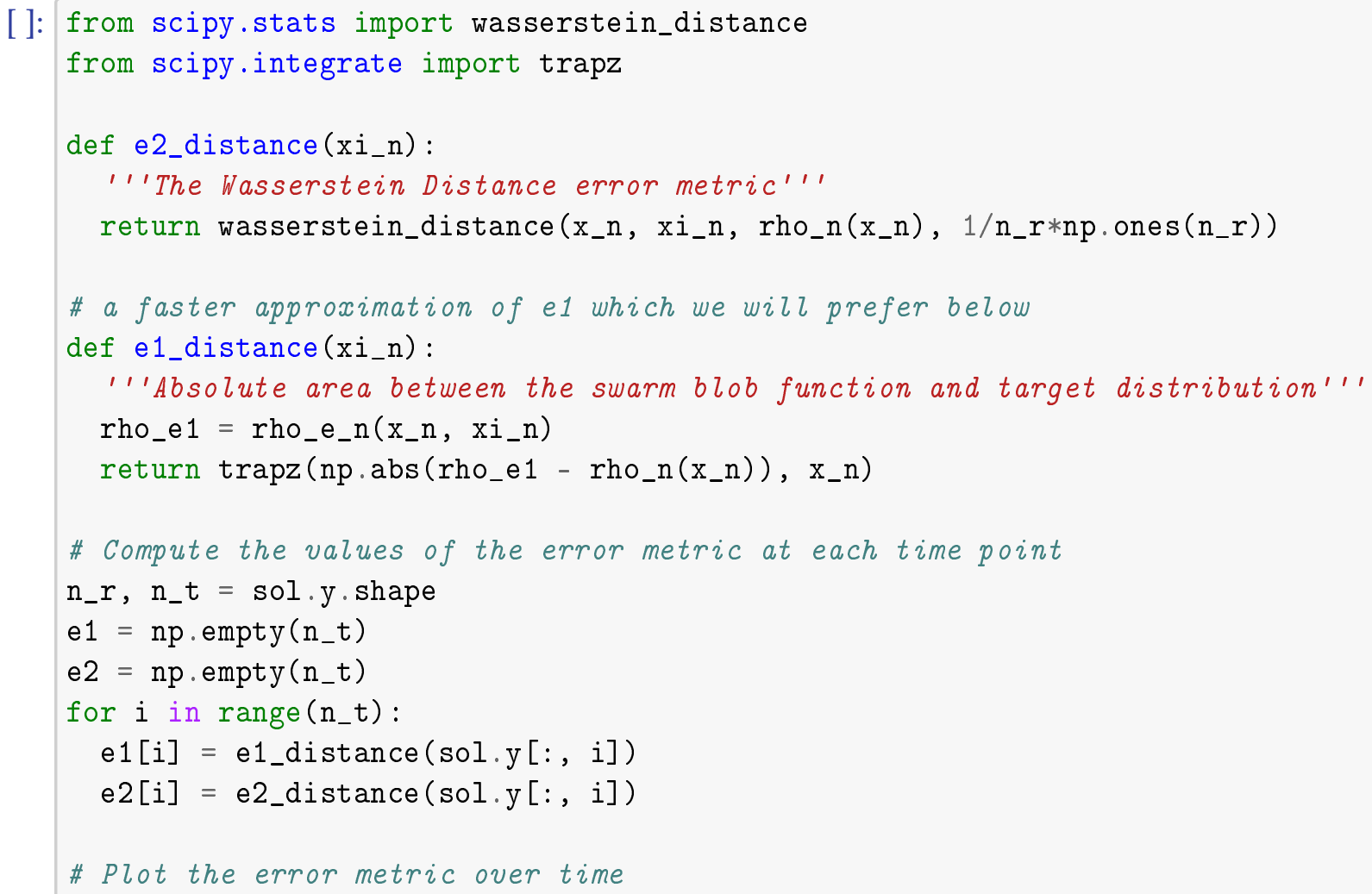



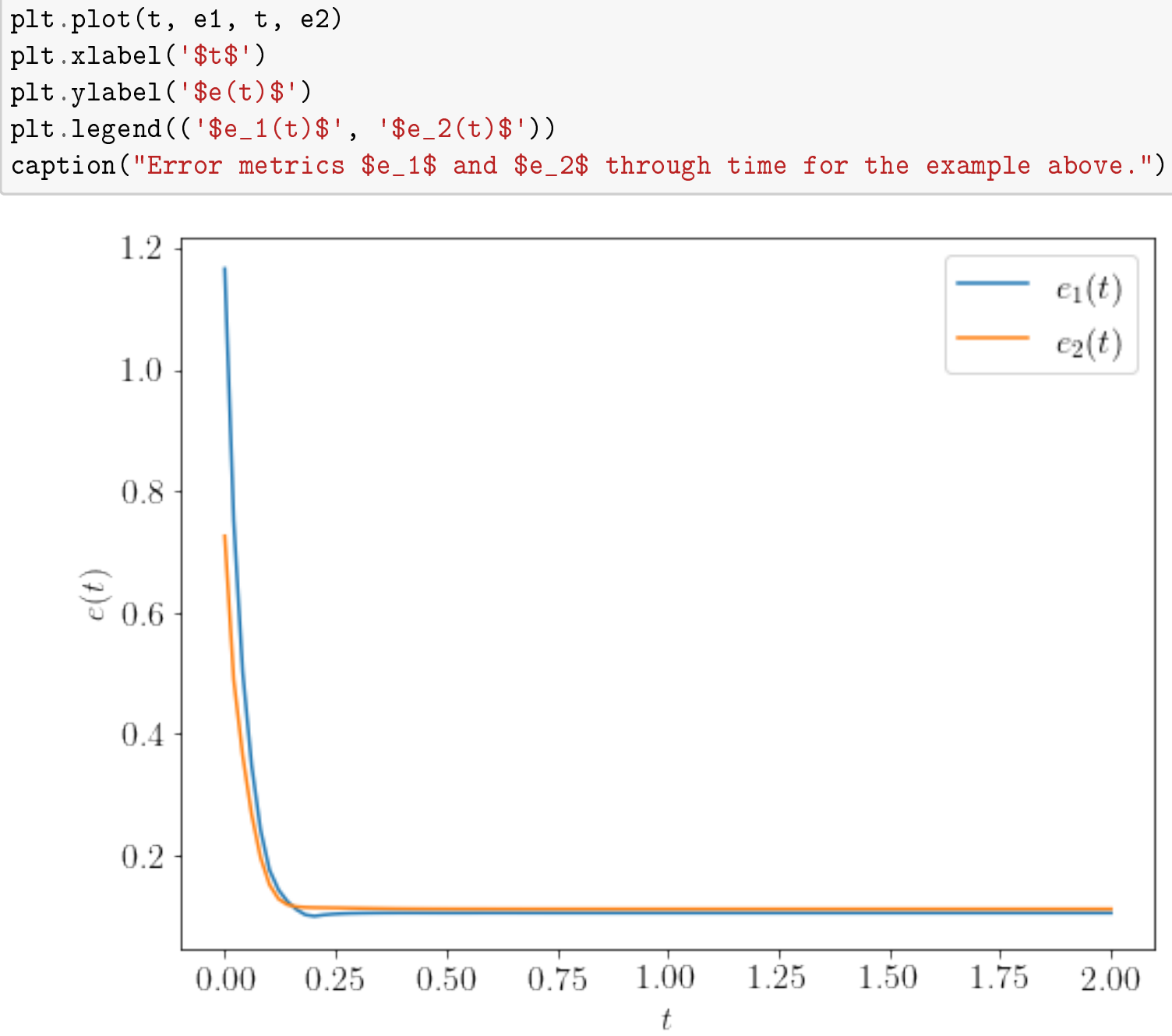

Figure 10. Error metrics $e_{1}$ and $e_{2}$ through time for the example above.

\subsection{Quantifying Speed}

Since the controller drives the system towards a steady state, we can quantify the speed of the controller using a conventional definition of settling time: the time after which the value of the error metric remains within $2 \%$ if its final value. Defining $e_{0}$ as the value of the error metric at the beginning of the simulation and $e_{\infty}$ as the value of the error metric at the end of the simulation, the value of the error metric at which the system is said to have settled is

$$
e_{\text {settle }}=e_{S S}+0.02\left(e_{0}-e_{s S}\right)
$$

and the settling time

$$
\begin{aligned}
& t_{\text {settle }}=\quad \max _{t} t \\
& \text { s.t. } e(t)=e_{\text {settle }} .
\end{aligned}
$$




\section{[]: from scipy.interpolate import interp1d}

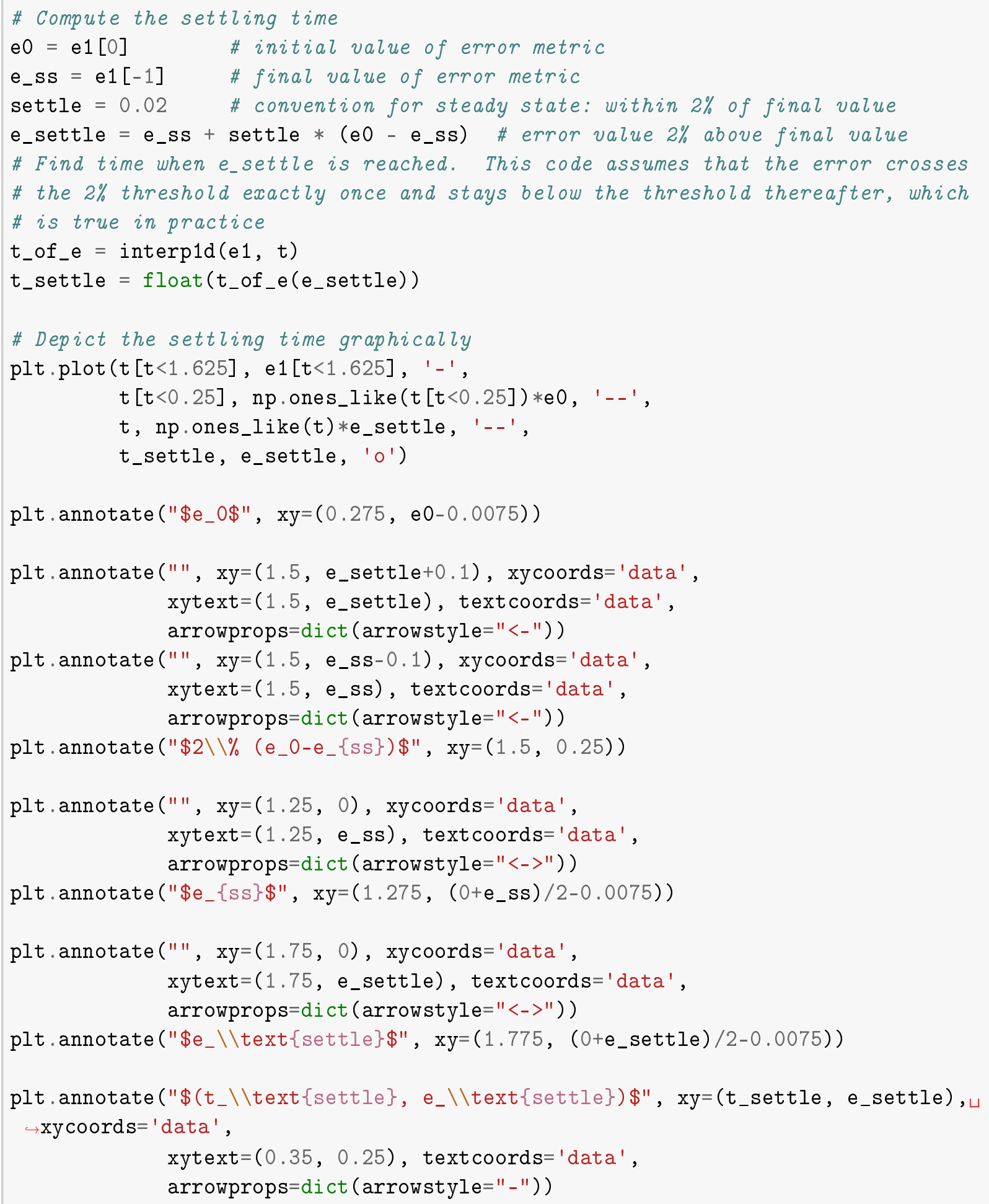



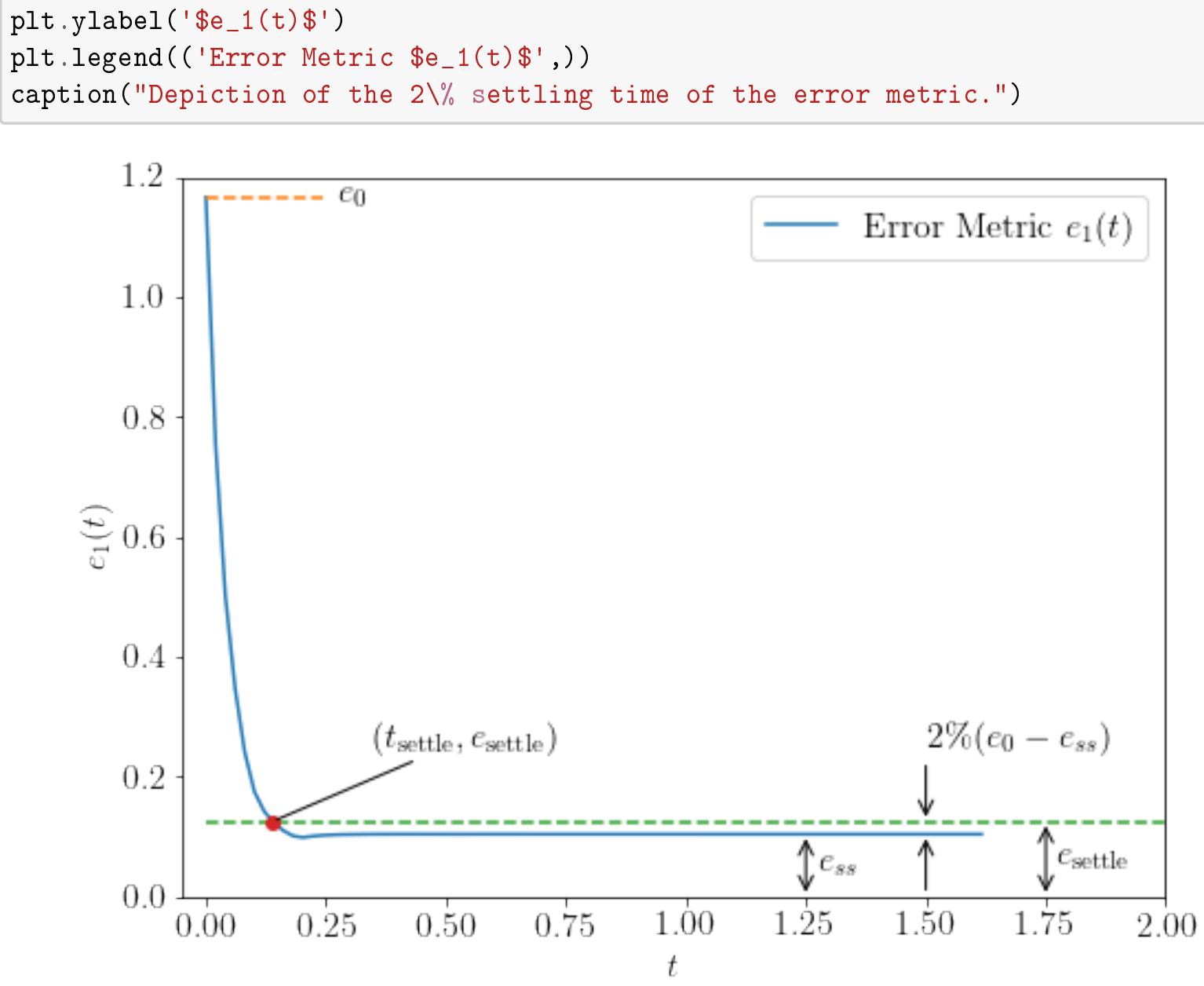

Figure 11. Depiction of the $2 \%$ settling time of the error metric.

\subsection{Optimal Swarm Configuration}

Note that it may not be possible, given swarm parameters such as the number of robots and each robot's radius of influence, to reduce the value of an error metric to zero. To better interpret whether the achieved value of an error metric is acceptable, we use numerical optimization to find the minimum possible value of the error metric.

[]: from scipy.optimize import minimize

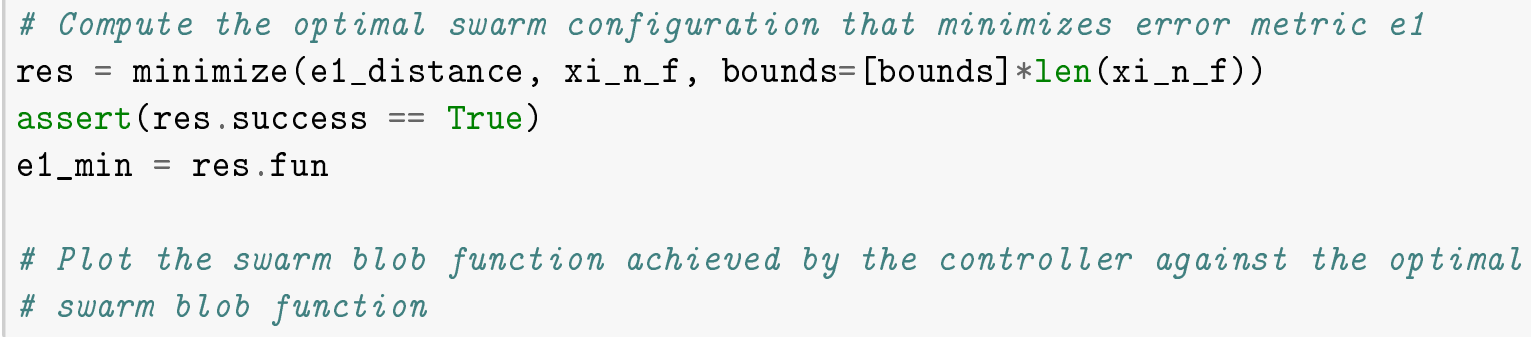



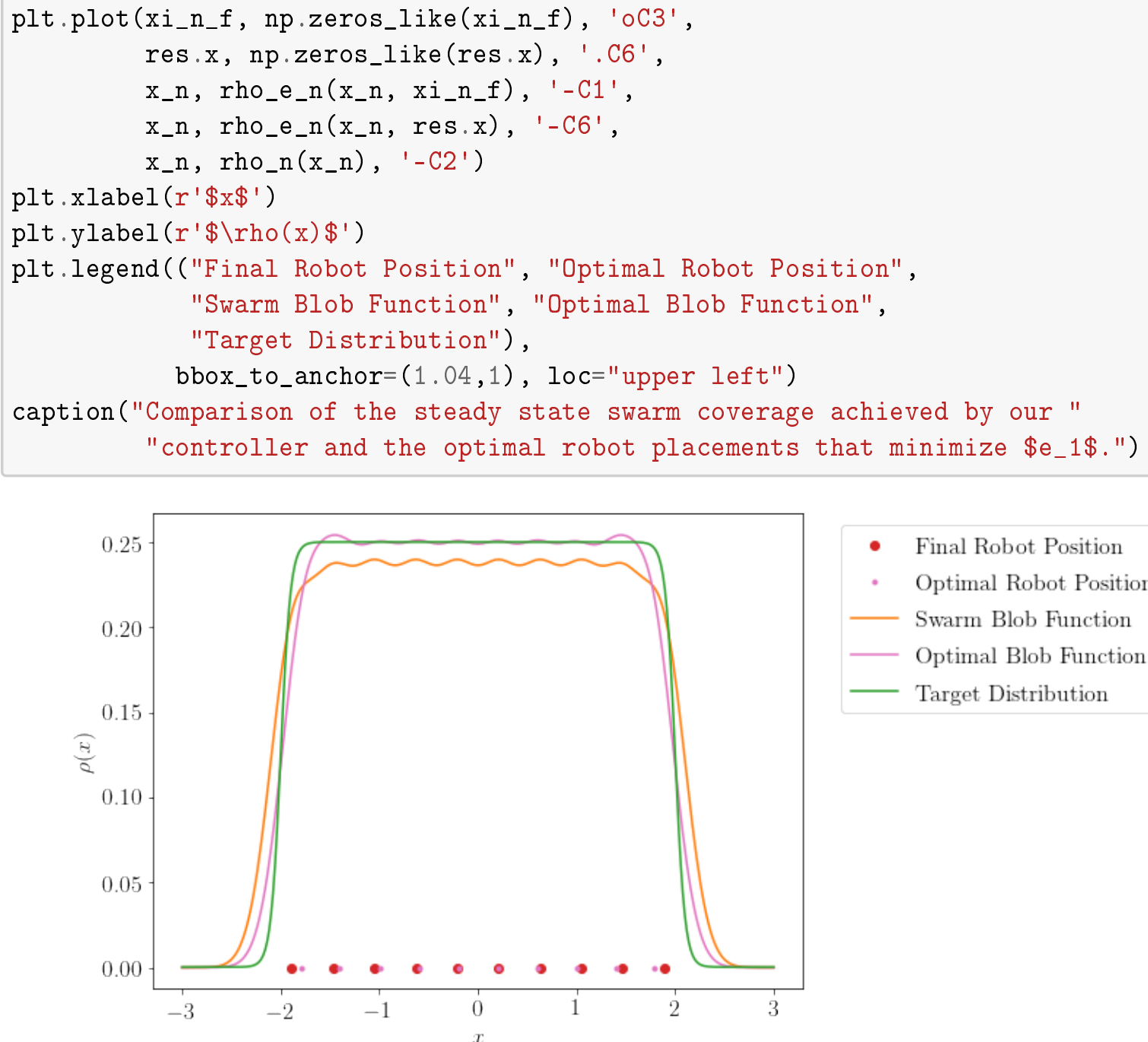

- Final Robot Position

- Optimal Robot Position

- Swarm Blob Function

- Optimal Blob Function

— Target Distribution

Figure 12. Comparison of the steady state swarm coverage achieved by our controller and the optimal robot placements that minimize $e_{1}$.

\subsection{Simulation Termination}

For a large swarm, the error metric is not very sensitive to position changes of individual robots, so it is not a reliable way to detect when the swarm distribution has stopped evolving and the simulation should end. Instead, we stop the simulation when the $L^{1}$-norm of the robot velocities have fallen below a threshold, which is manually chosen to maintain accuracy without requiring unnecessarily long simulation.

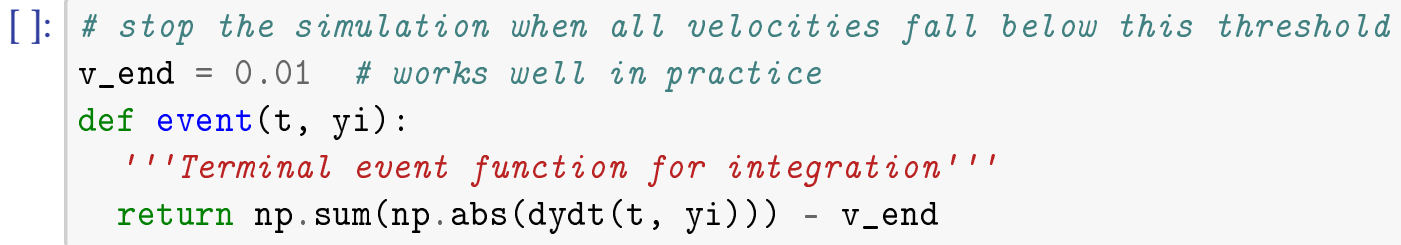




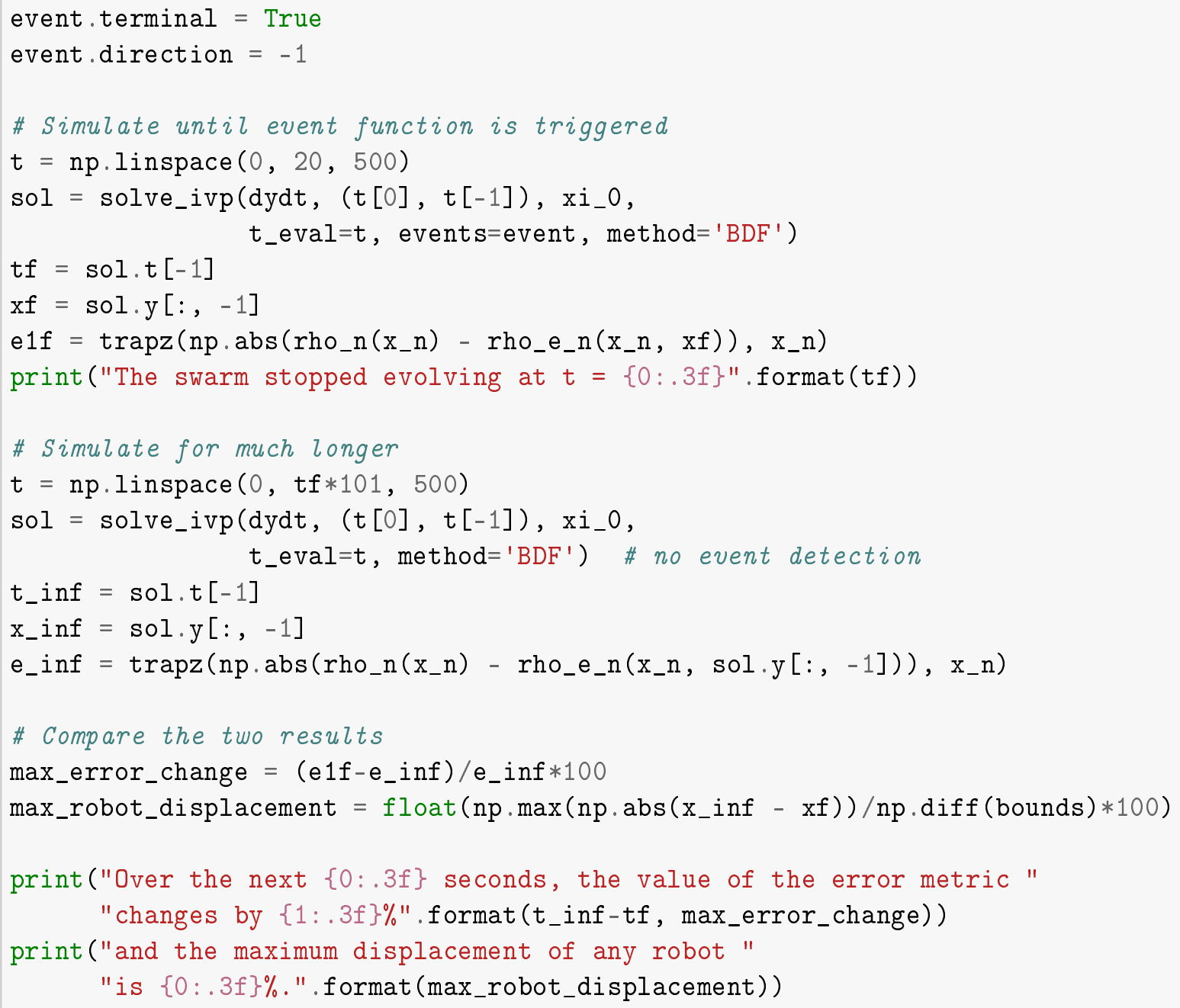

The swarm stopped evolving at $t=1.082$

Over the next 108.216 seconds, the value of the error metric changes by $-0.000 \%$ and the maximum displacement of any robot is $0.008 \%$.

\subsection{Independent Variables}

We are currently exploring the effects of the following independent variables on the accuracy and speed of our control laws.

\subsubsection{Target Distribution}

The example above is for a uniform target distribution, but the control law can also guide the swarm to achieve a prescribed non-uniform coverage. How do the properties of the target distribution, such as smoothness, affect controller performance?

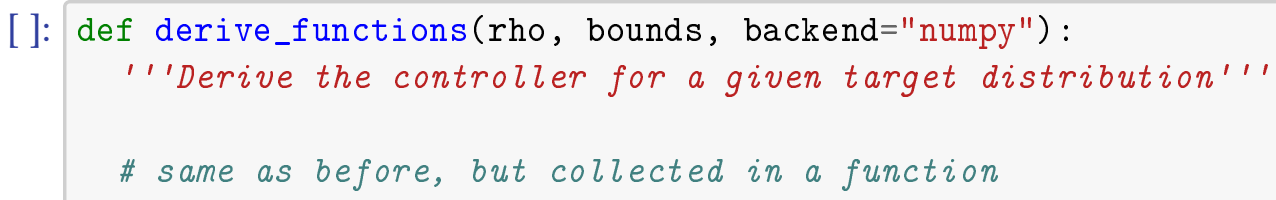



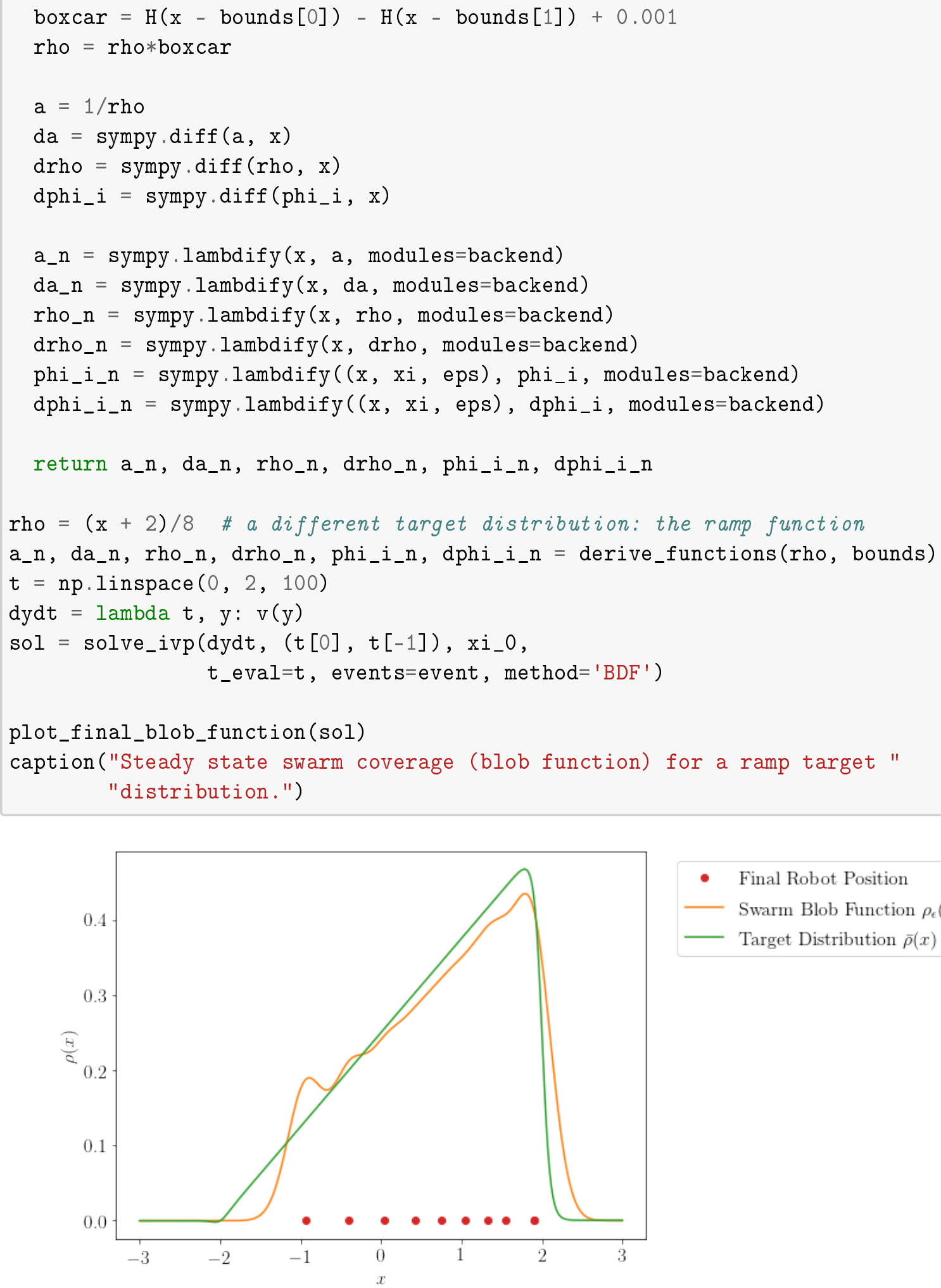

- Final Robot Position

Swarm Blob Function $\rho_{\epsilon}(x)$

Target Distribution $\bar{\rho}(x)$

Figure 13. Steady state swarm coverage (blob function) for a ramp target distribution. 


\subsubsection{Initial Swarm Configuration}

The example above begins with all robots clustered about the origin. Intuitively the initial configuration will affect the settling time, but how much? Does it affect the final configuration?
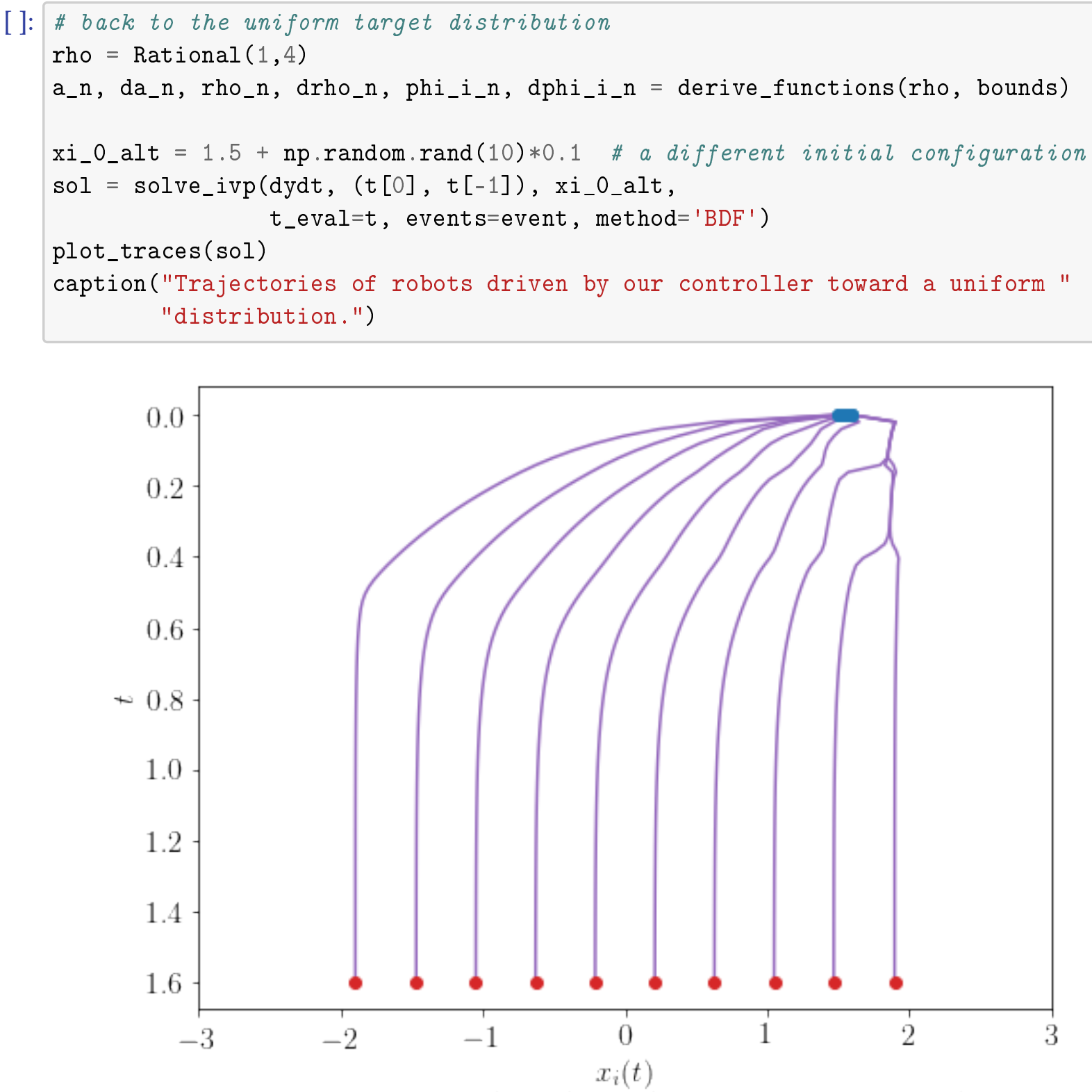

Figure 14. Trajectories of robots driven by our controller toward a uniform distribution.

\subsubsection{Robot Speed Limits}

In the example above, there were no limitations on a robot's speed. Without a speed limit, measuring the settling time is not very meaningful, as any controller can be trivially modified to achieve 
any desired settling time: simply scale all robot velocities by a constant factor. However, these controllers tend to work even when a speed limit is imposed on the robots. Which performs best with this limitation?

[]: def $\operatorname{dydt}(t, y)$ :

"Calculate robot velocities subject to a speed limit"'

$\mathrm{v}_{\text {_max }}=1$ \# speed limit

$\mathrm{v}_{\text {_prescribed }}=\mathrm{v}(\mathrm{y})$

v_actual $=v_{-}$prescribed

$v_{-}$actual [v_actual $\left.>v_{-} \max \right]=v_{-} \max$

$v_{-}$actual [v_actual $\left.<v_{-} \max \right]=-v_{-} \max$

return v_actual

sol = solve_ivp $\left(\right.$ dydt, $(t[0], t[-1]), x i \_0$,

$t_{-}$eval=t, events=event, $\operatorname{method}={ }^{\prime} R K 45^{\prime}$ )

plot_traces ( $\mathrm{sol})$

caption("Trajectories of speed-limited robots governed by our controller.")

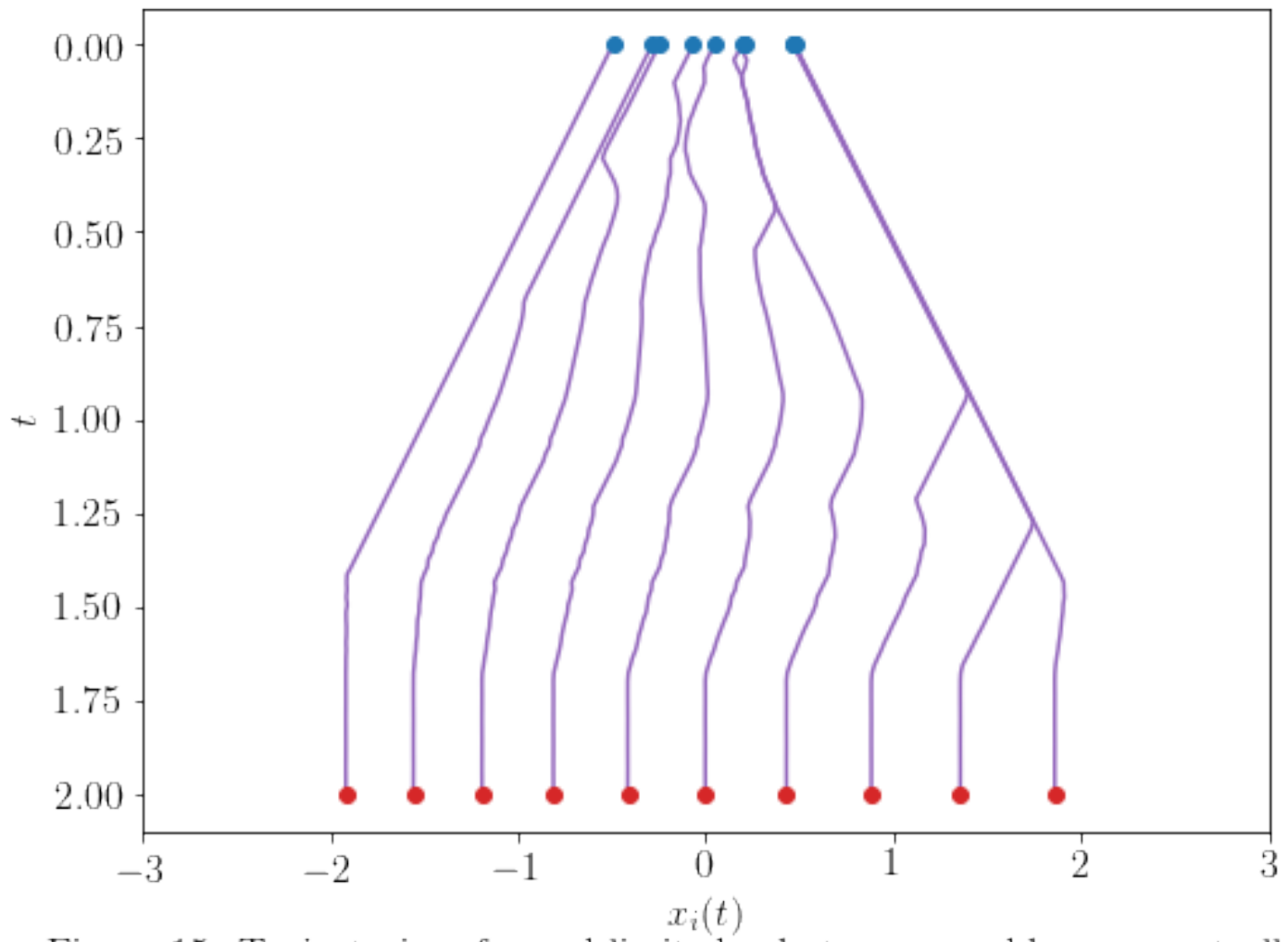

Figure 15. Trajectories of speed-limited robots governed by our controller. 


\subsubsection{Mollifier Function $\phi$ and Influence Radius $\epsilon$}

Previously, we used the Gaussian function as the mollifier $\phi$ to describe how the robot's influence diminishes as a function of distance. The Gaussian function is always nonzero, that is, it has noncompact support. Real robots, however, will have limited sensing range and influence, so we are interested in the use of mollifiers with compact support. If robots are not able to sense the influence of all others in the swarm, will the swarm still converge to the desired distribution? Do some mollifiers, such as those used as kernels in nonparametric statistical estimation, perform better than others?

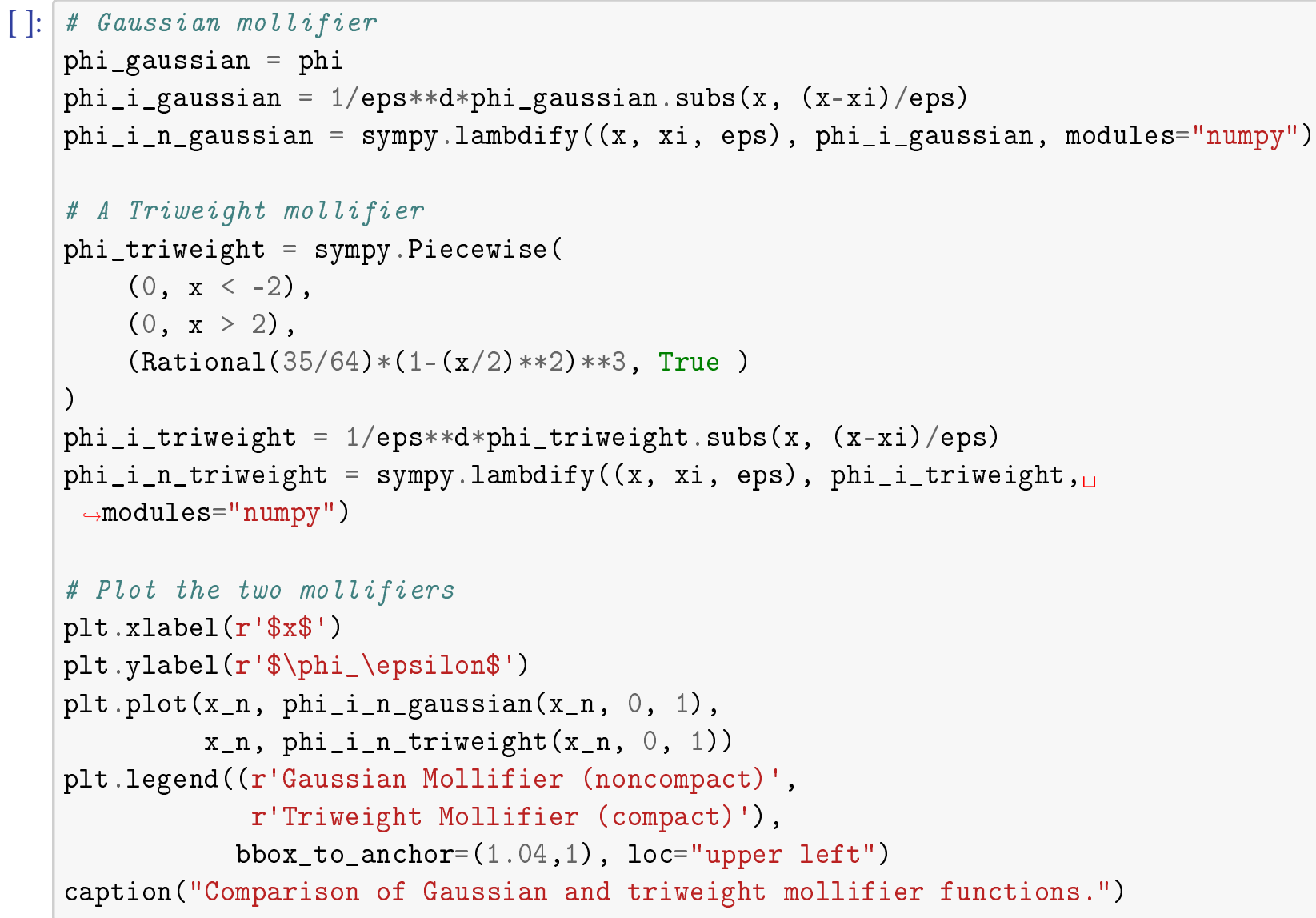




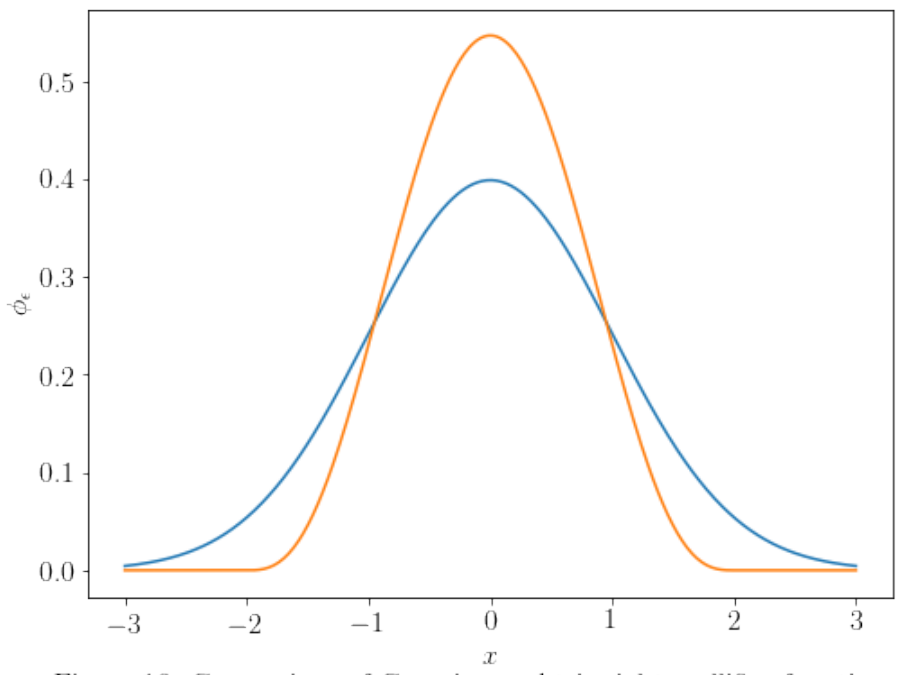

Gaussian Mollifier (noncompact)

Triweight Mollifier (compact)

Figure 16. Comparison of Gaussian and triweight mollifier functions
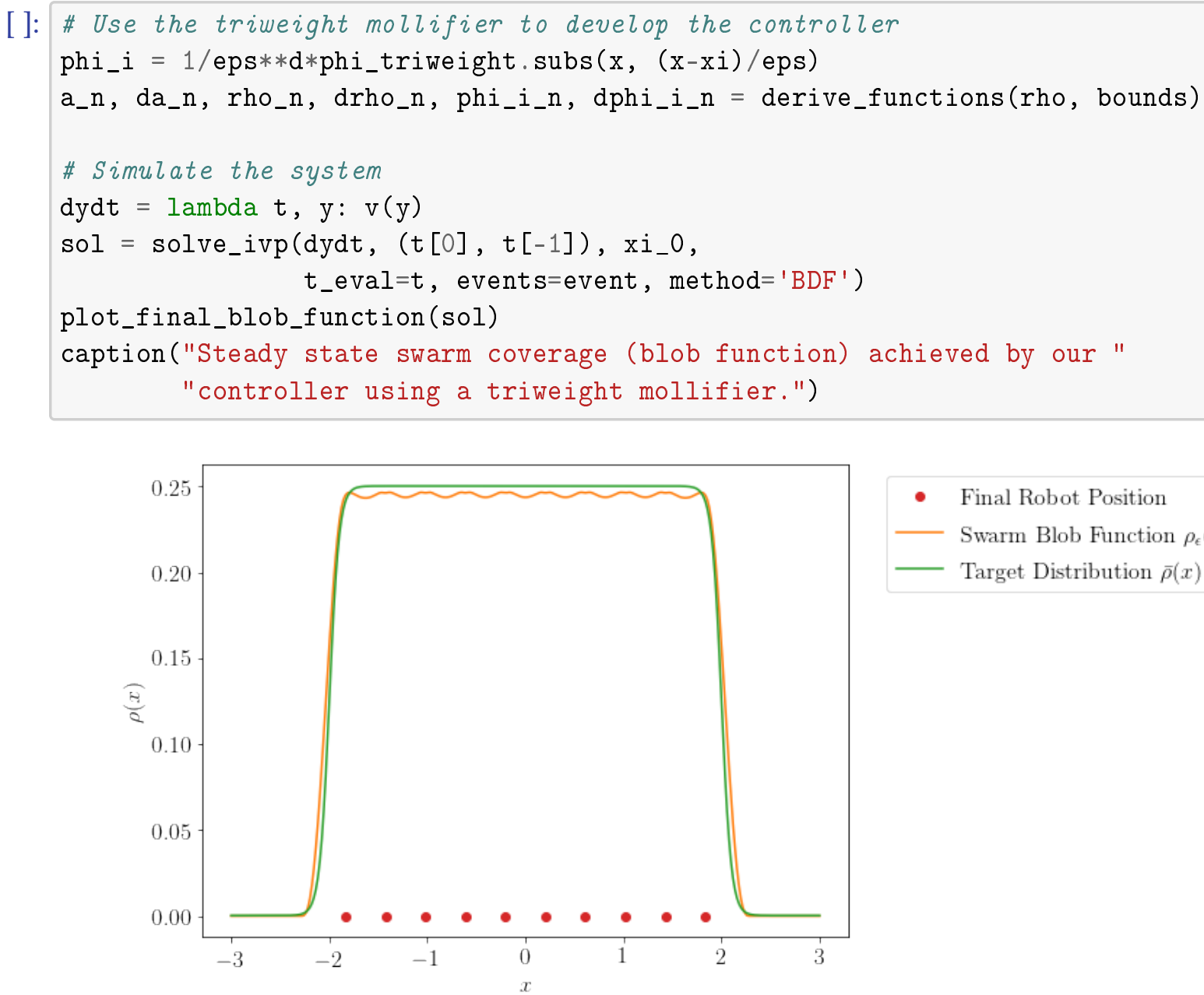

- Final Robot Position

Swarm Blob Function $\rho_{\epsilon}(x)$

Target Distribution $\bar{\rho}(x)$

Figure 17. Steady state swarm coverage (blob function) achieved by our controller using a triweight mollifier. 


\subsubsection{Swarm Size}

As the number of robots increases, the ability of the swarm to achieve the desired distribution should improve, but at what rate? How many robots are needed to achieve a desired accuracy?

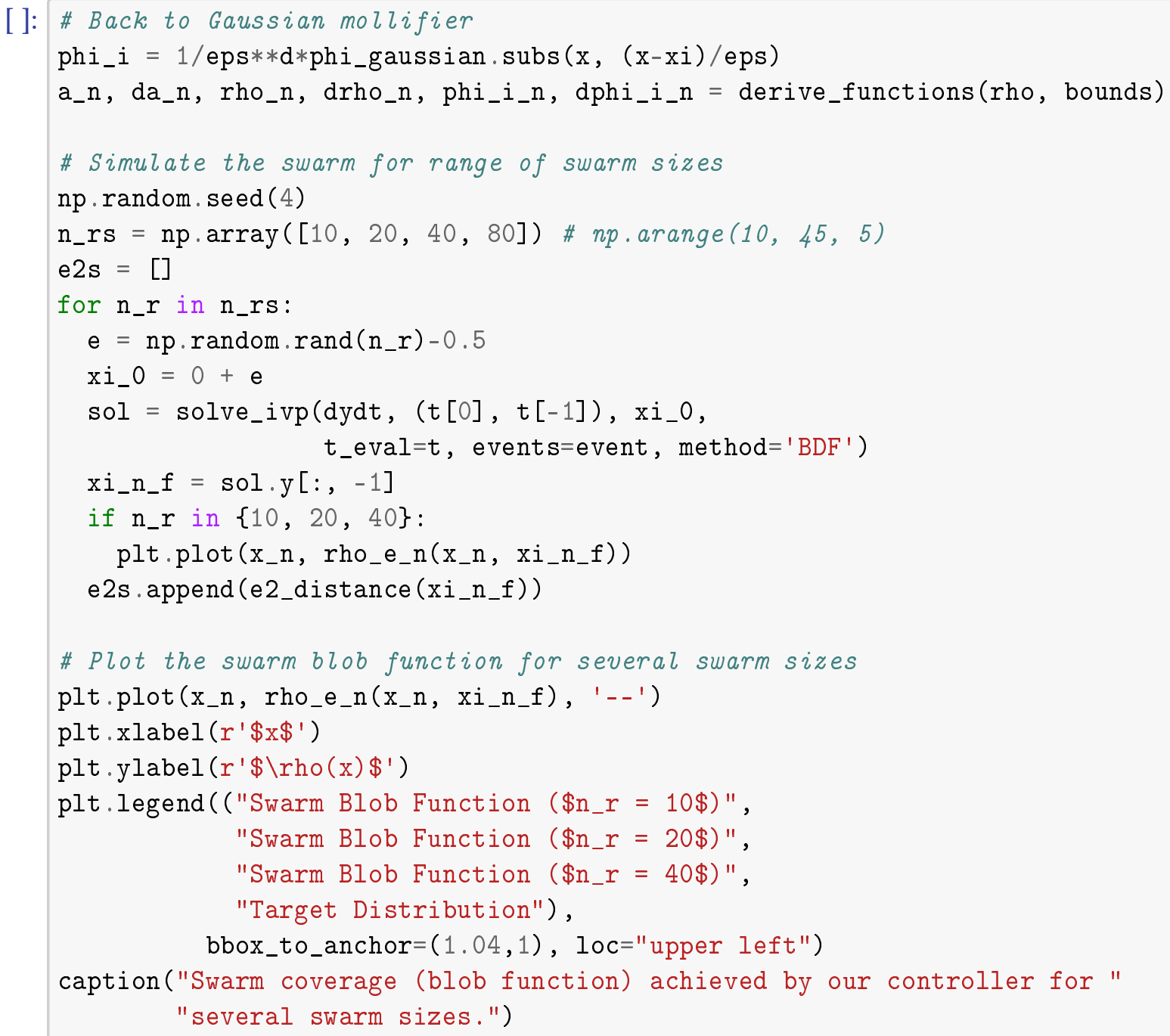




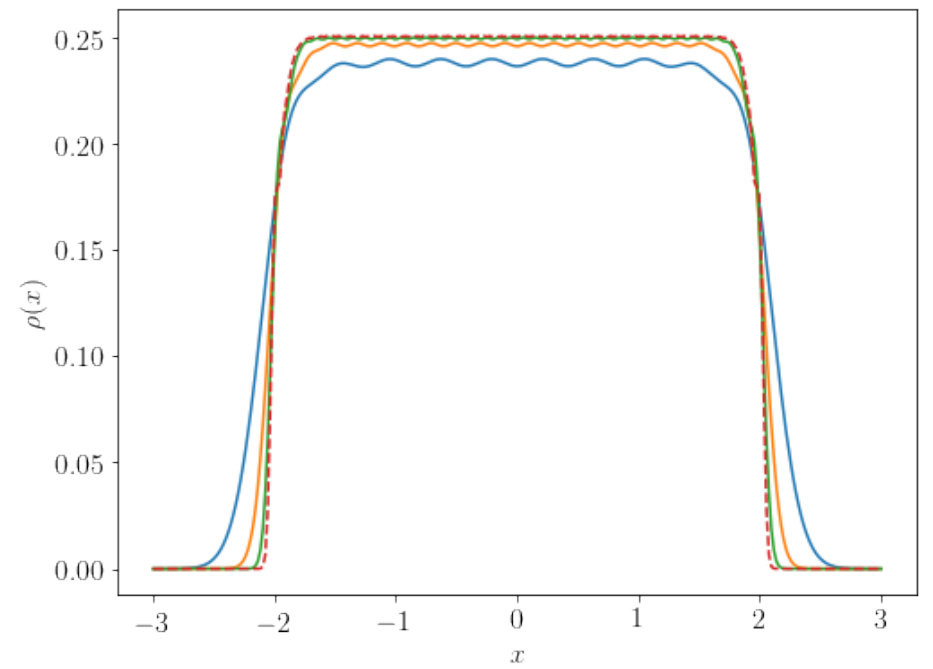

- Swarm Blob Function $\left(n_{r}=10\right)$

- Swarm Blob Function $\left(n_{r}=20\right)$

Swarm Blob Function $\left(n_{r}=40\right)$

---- Target Distribution

Figure 18. Swarm coverage (blob function) achieved by our controller for several swarm sizes.

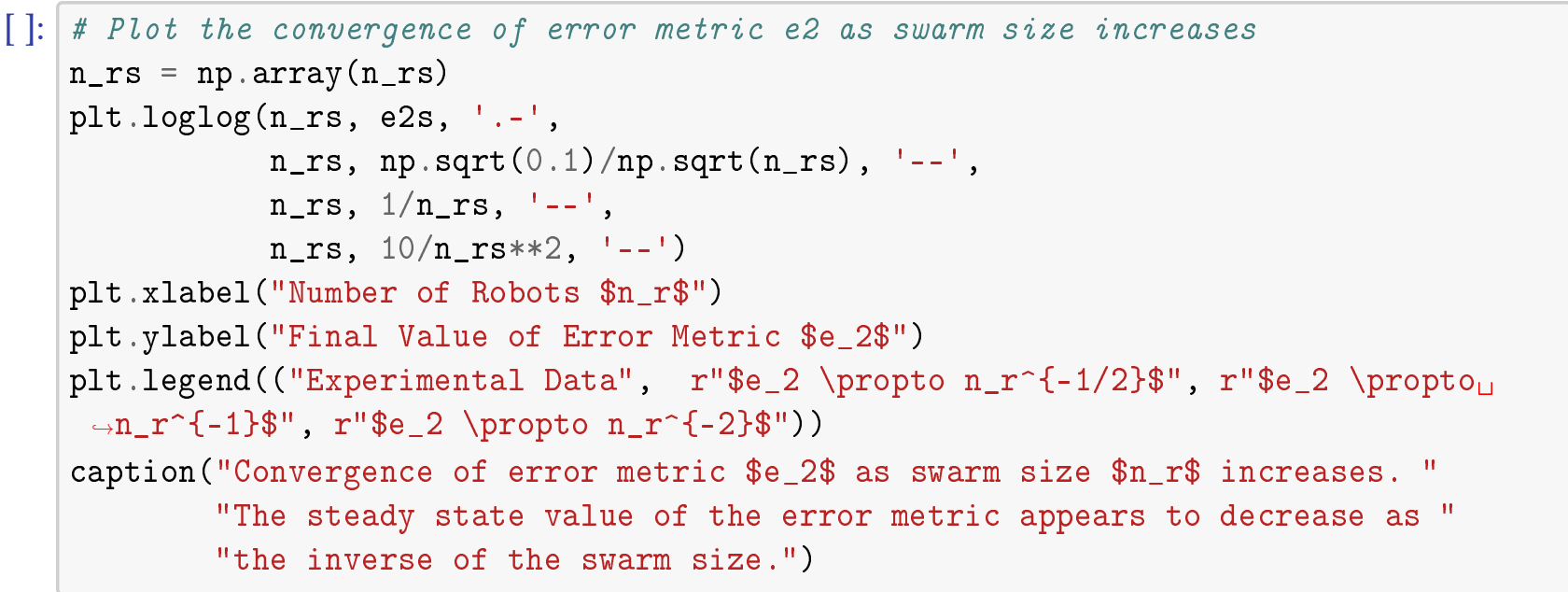




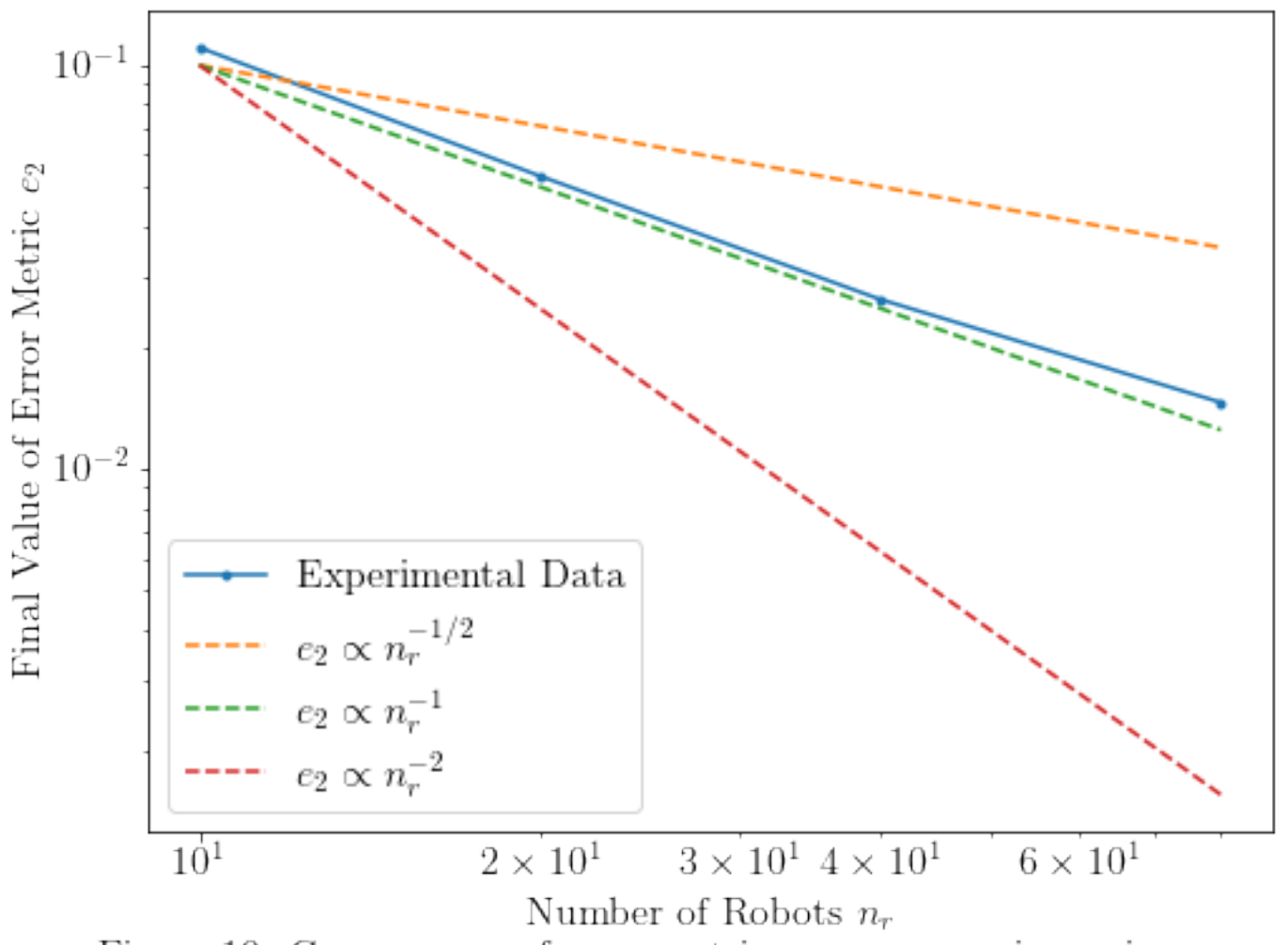

Figure 19. Convergence of error metric $e_{2}$ as swarm size $n_{T}$ increases. The steady state value of the error metric appears to decrease as the inverse of the swarm size.

Numerical results will guide analytical study of the mathematical properties of these controllers.

\section{Computational Discussion}

\subsection{Computation Complexity of Simulation}

We must simulate large swarms to investigate the effect of swarm size on controller speed and accuracy, but without care, this becomes very computationally expensive. Based on the form of (3), we would expect the computational complexity to be at least $O\left(n_{r}^{2}\right)$ : in order to calculate the velocity $v_{i}$ for each of $n_{r}$ robots $i$, we must sum contributions from $n_{r}$ robots $j$.

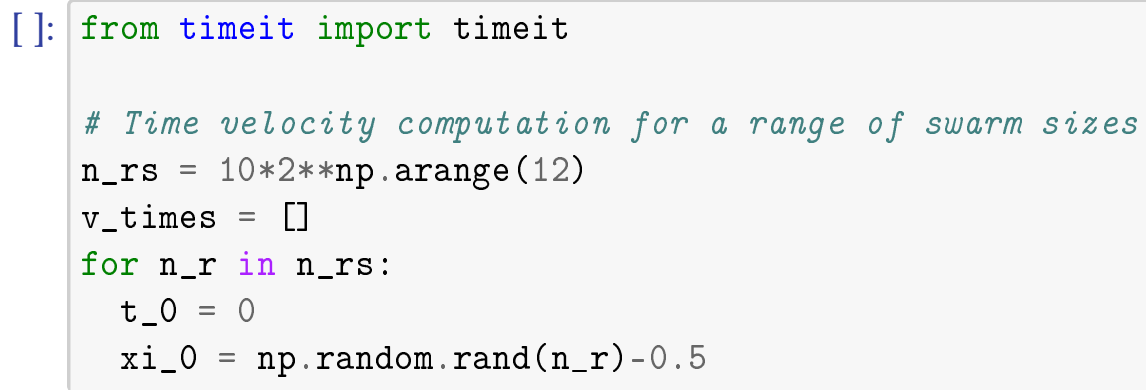



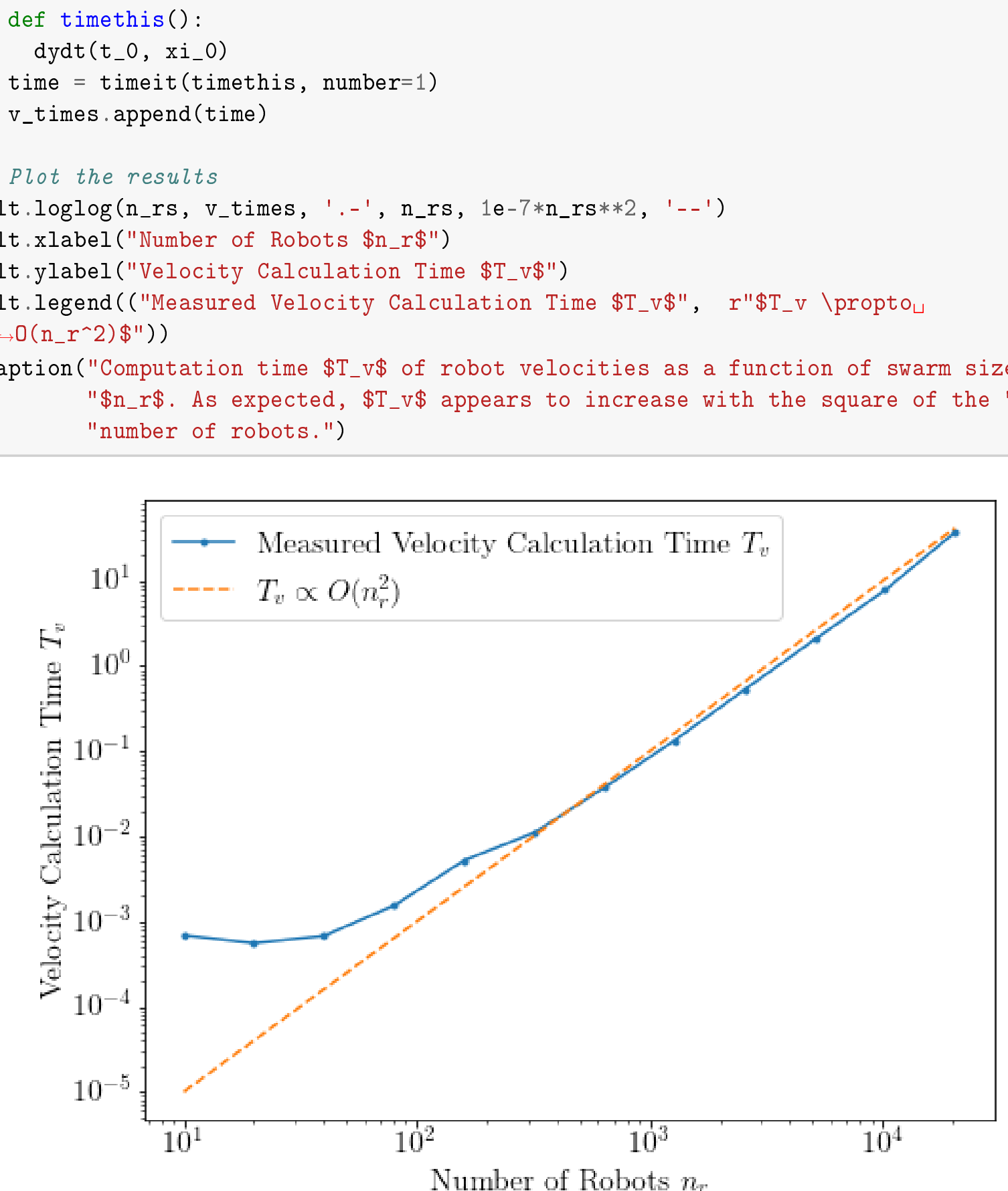

Figure 20. Computation time $T_{v}$ of robot velocities as a function of swarm size $n_{\tau}$. As expected, $T_{v}$ appears to increase with the square of the number of robots.

However, total simulation time increases even faster than that. 


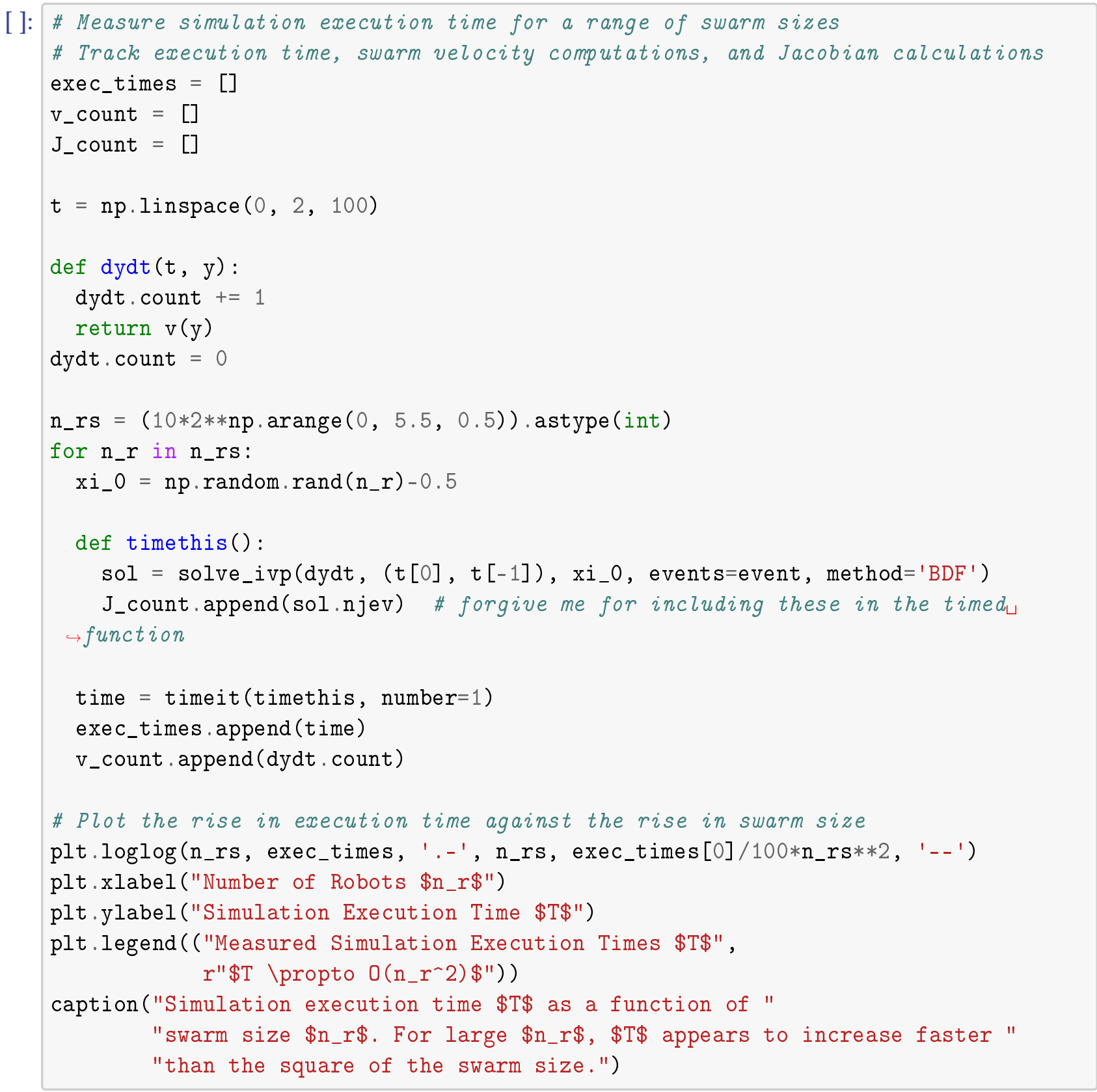




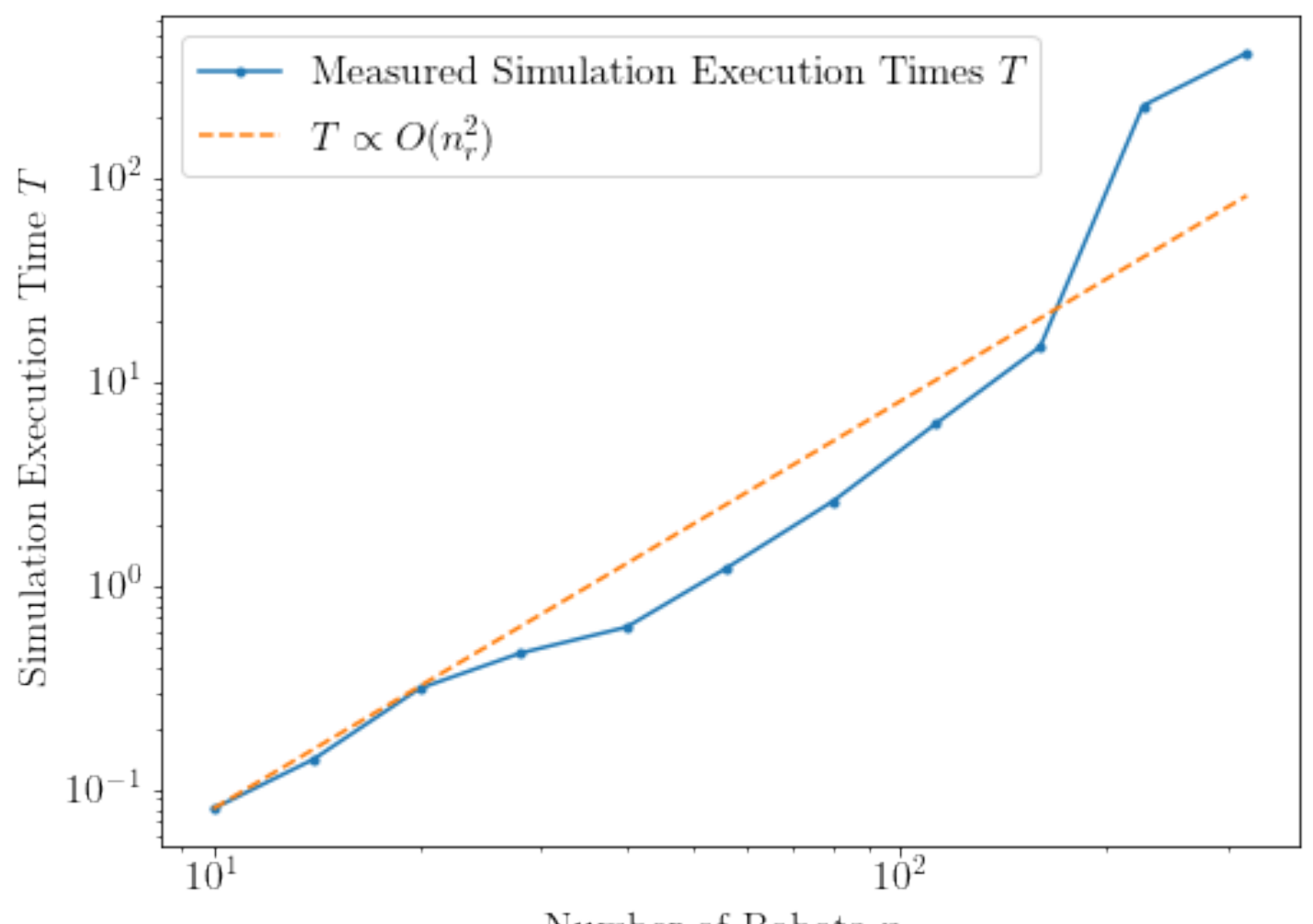

Number of Robots $n_{T}$

Figure 21. Simulation execution time $T$ as a function of swarm size $n_{T}$.

For large $n_{T}, T$ appears to increase faster than the square of the swarm size.

The increase in slope at the end is not noise; rather, the execution time $T$ is increasing faster than $O\left(n_{r}^{2}\right)$ for $n_{r}$ sufficiently large. This additional computational complexity comes from a few factors, the most significant of which is that the number of velocity function executions required also increases with the swarm size.

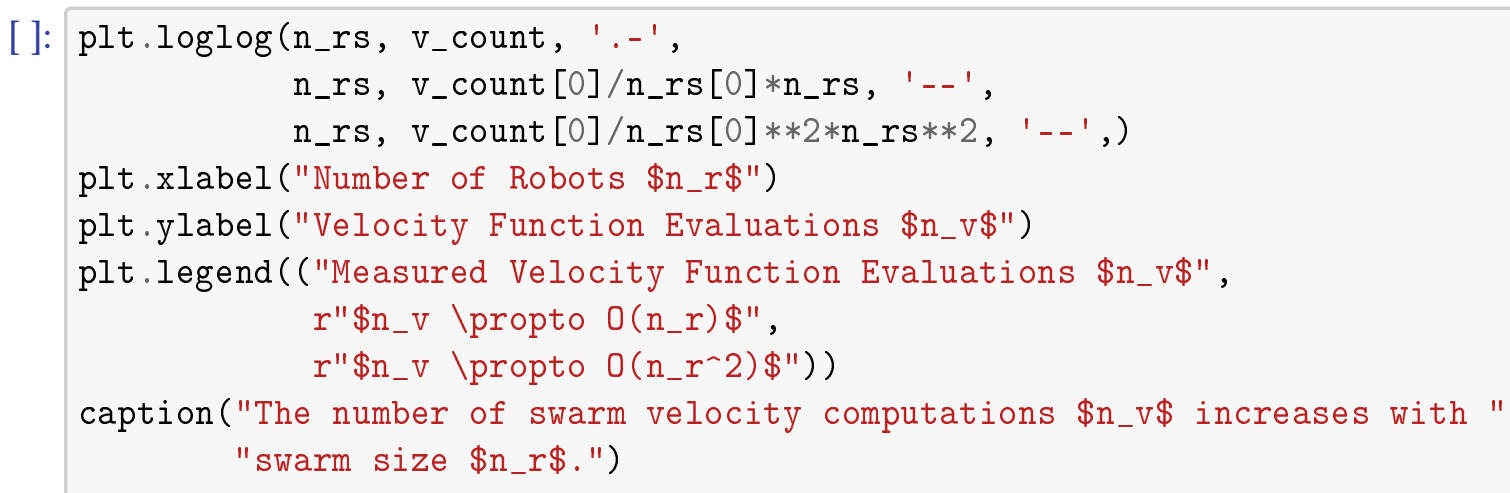




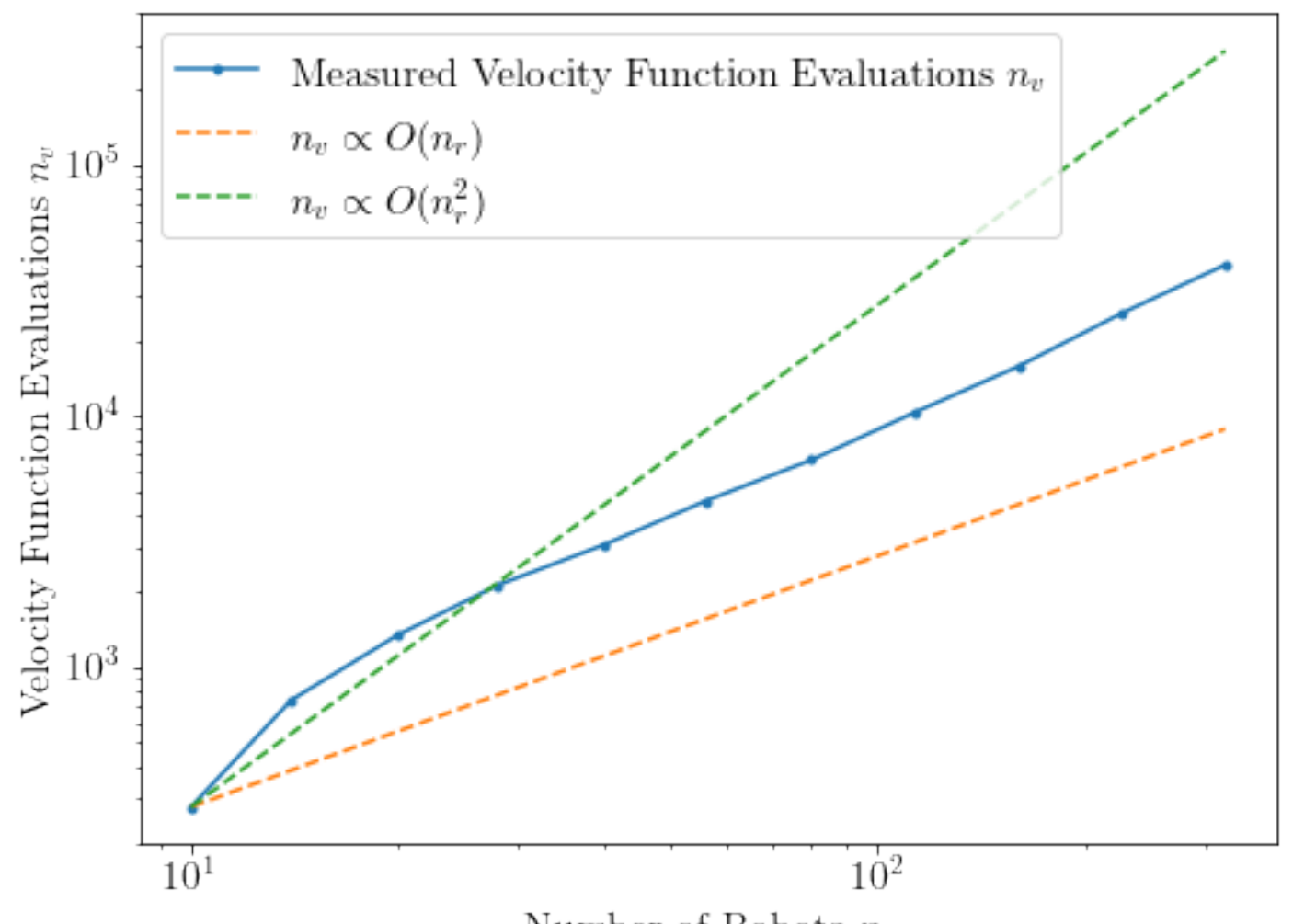

Figure 22. The number of swarm velocity computations $n_{v}$ increases with swarm size $n_{r}$.

To see why, we need to address the choice of ODE solver. In most simulations above, the choice has been 'BDF' because the ODEs are known to be stiff. It is quite clear that an implicit solver is the appropriate choice when we compare the performance of all the solvers.

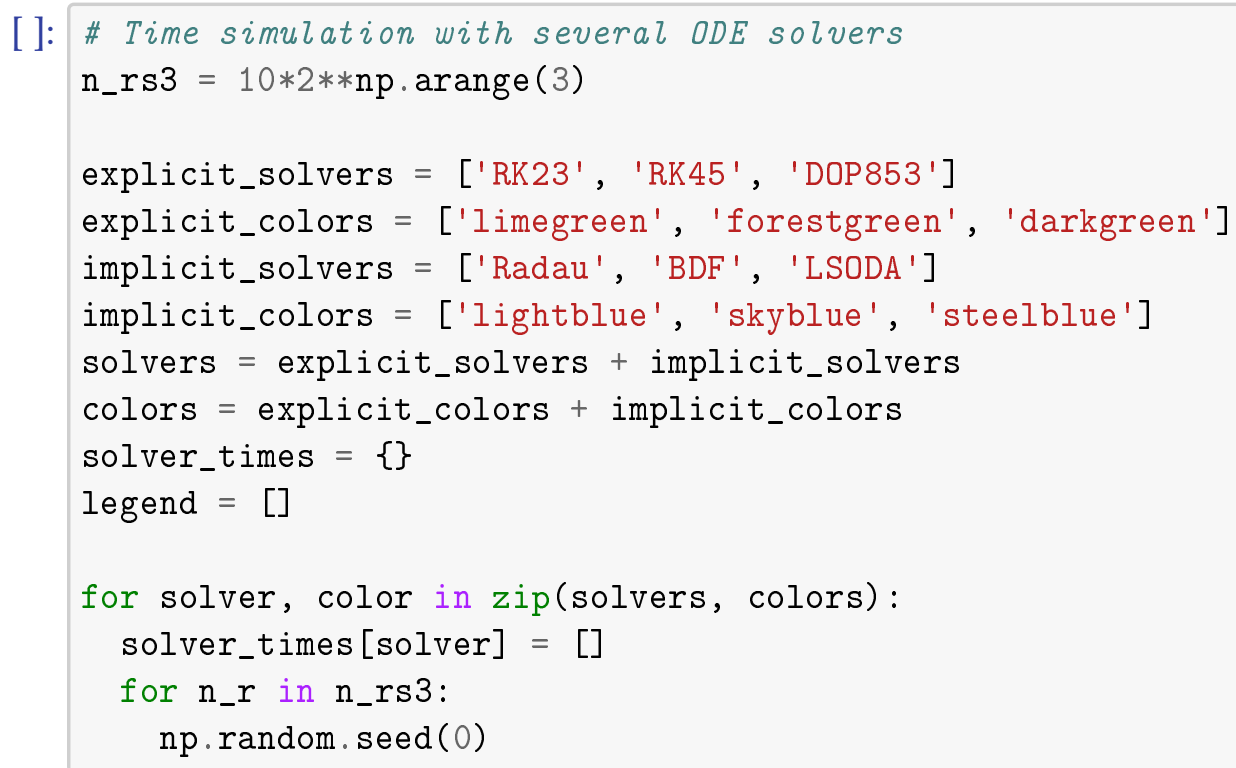



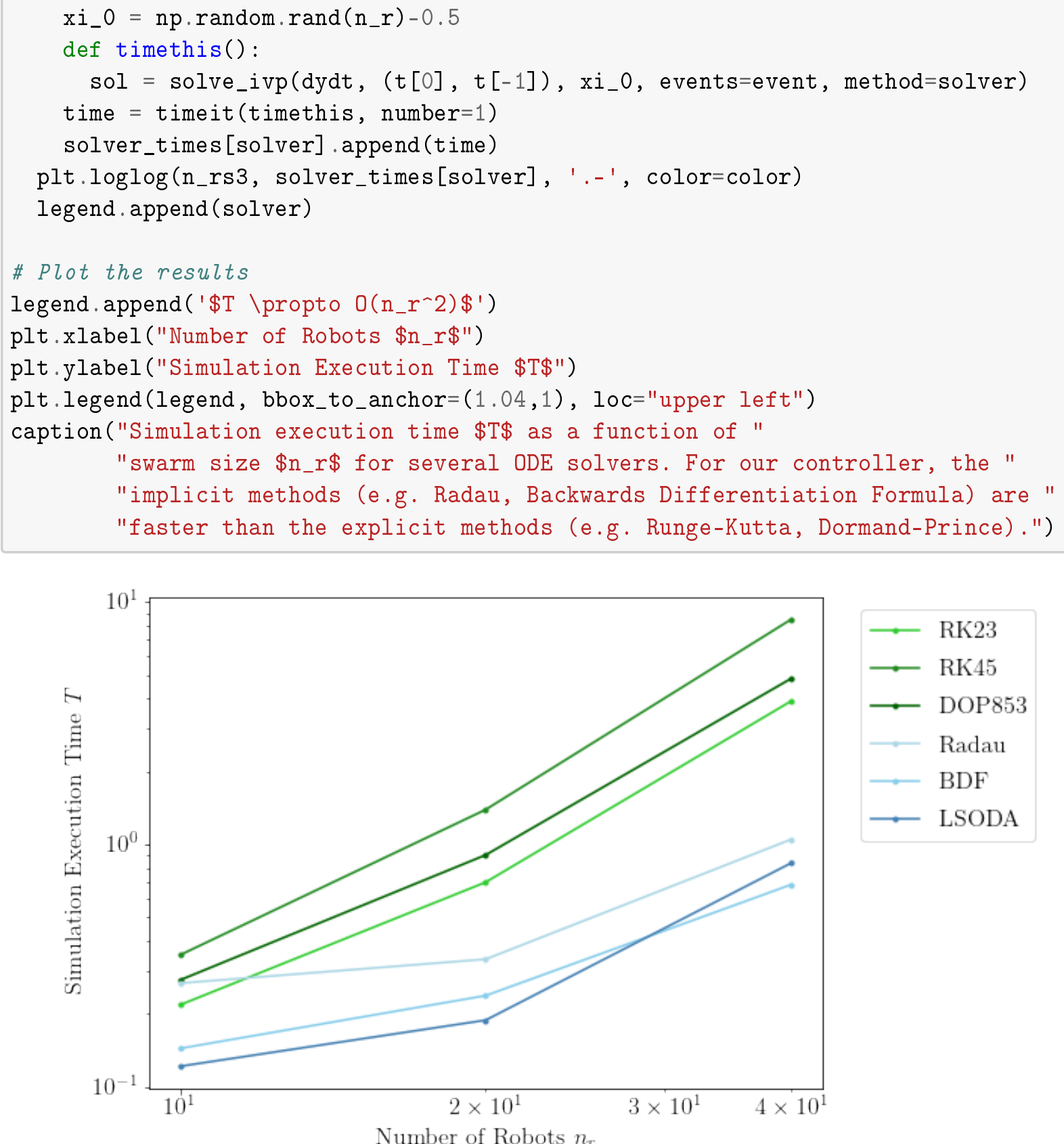

Figure 23. Simulation execution time $T$ as a function of swarm size $n_{r}$ for several ODE solvers. For our controller, the implicit methods (e.g. Radau, Backwards Differentiation Formula) are faster than the explicit methods (e.g. Runge-Kutta, Dormand-Prince).

The explicit solvers (green) are much slower than the implicit solvers(blue), and the gap appears to grow as the swarm size increases. We will continue our discussion assuming use of the BDF solver, as that has typically been the fastest in practice, but the discussion below applies to all of the implicit solvers.

Because BDF is an implicit solver, it needs to solve an equation $f\left(x\left(t_{k}\right), x\left(t_{k+1}\right)\right)=0$ for $x\left(t_{k+1}\right)$ 
at each time step $t_{k}$, and to do so it must evaluate the Jacobian of $f$, that is, the matrix of partial derivatives $\frac{\partial f_{i}}{\partial x_{j}}$. As implemented above, solve_ivp does this using finite differences, which involves evaluating the robot velocities $O\left(n_{r}\right)$ times. As discussed above, evaluating the robot velocities is itself an $O\left(n_{r}^{2}\right)$ operation according to (3). Hence, the computational complexity is at least $O\left(n_{r}^{3}\right)$.

Fortunately, the number of Jacobian evaluations seems to rise relatively slowly with the swarm size.
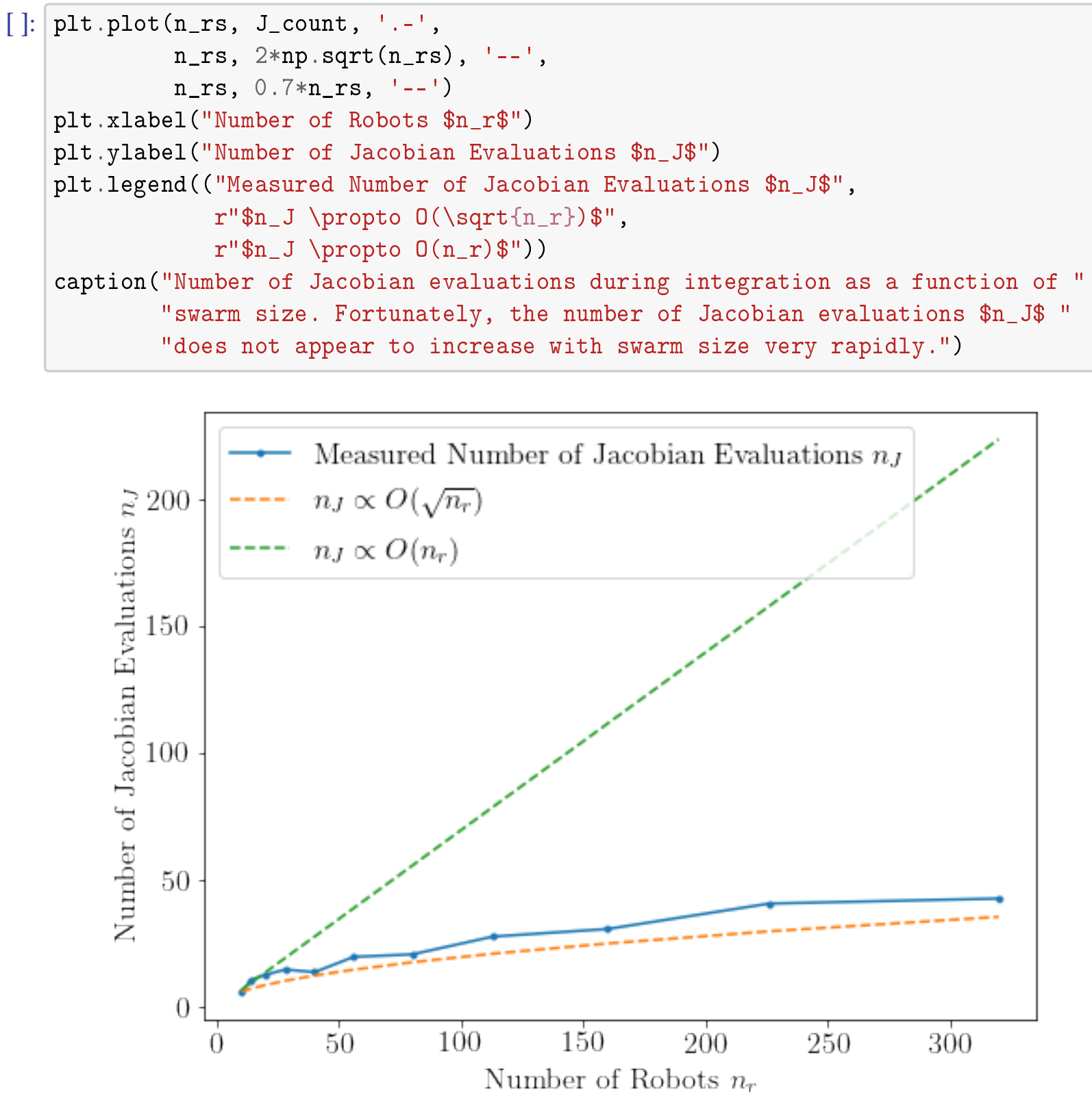

Figure 24. Number of Jacobian evaluations during integration as a function of swarm size. Fortunately, the number of Jacobian evaluations $n_{J}$ does not appear to increase with swarm size very rapidly. 


\subsection{Simulation Speed Improvements}

Now that we understand why the execution takes so long, we can begin to address it.

\subsubsection{NumExpr}

Using NumExpr instead of NumPy as the array math backend provides some relief when multiple CPU cores are available.

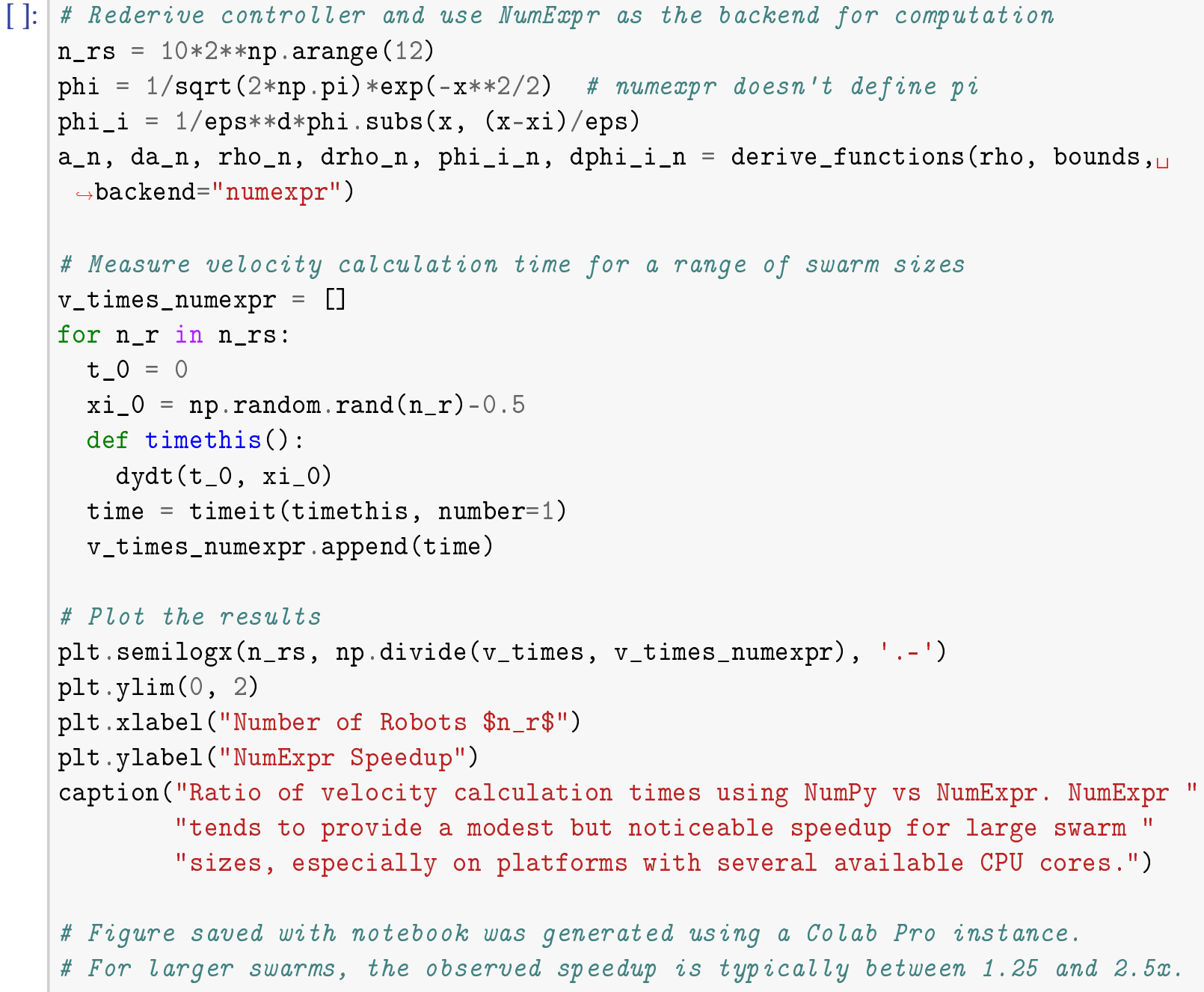




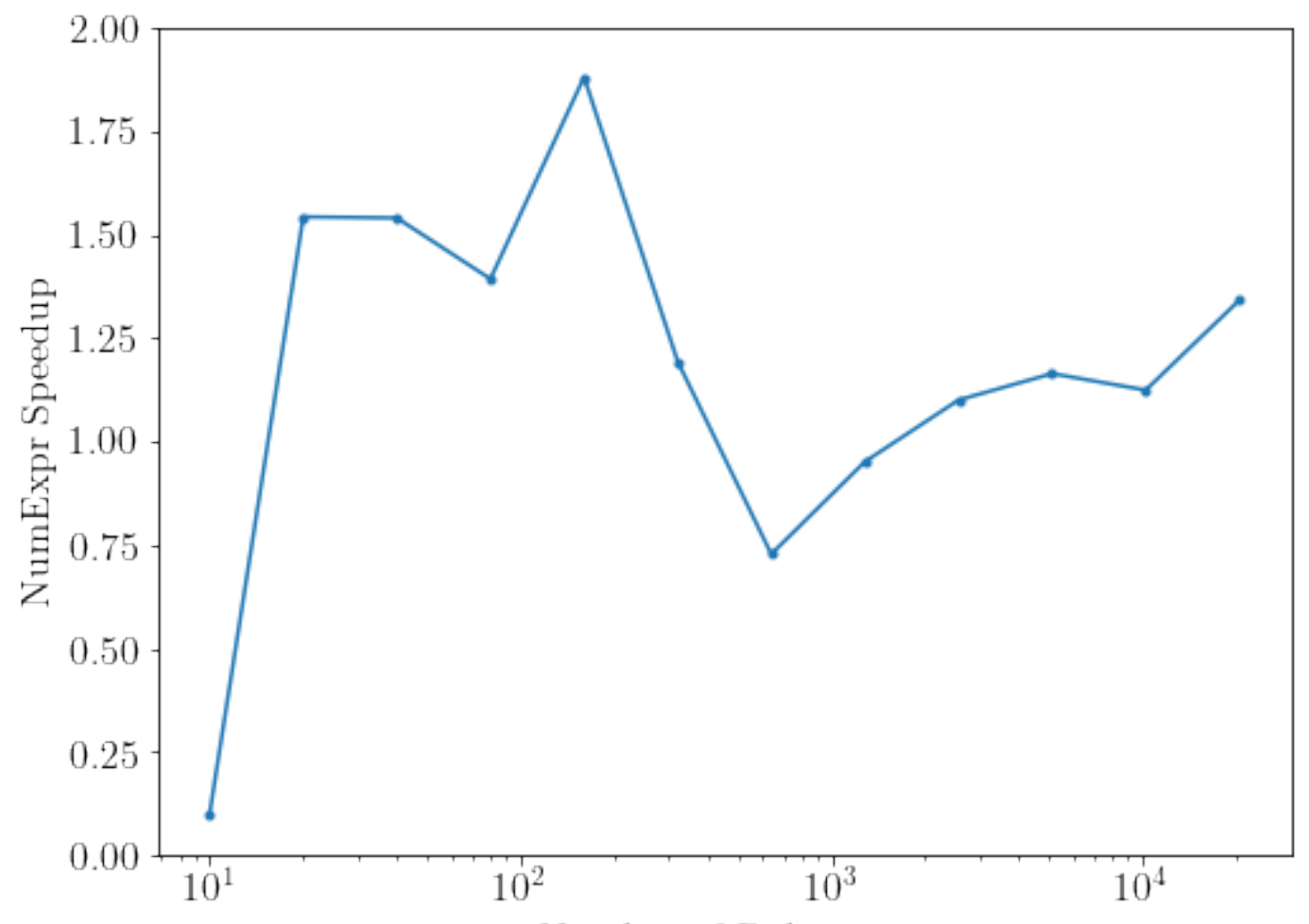

Number of Robots $n_{T}$

Figure 25. Ratio of velocity calculation times using NumPy vs NumExpr. NumExpr tends to provide a modest but noticeable speedup for large swarm sizes, especially on platforms with several available CPU cores.

\subsubsection{CuPy}

Alternatively, use of a GPU to perform the velocity calculations (via CuPy) is very promising.

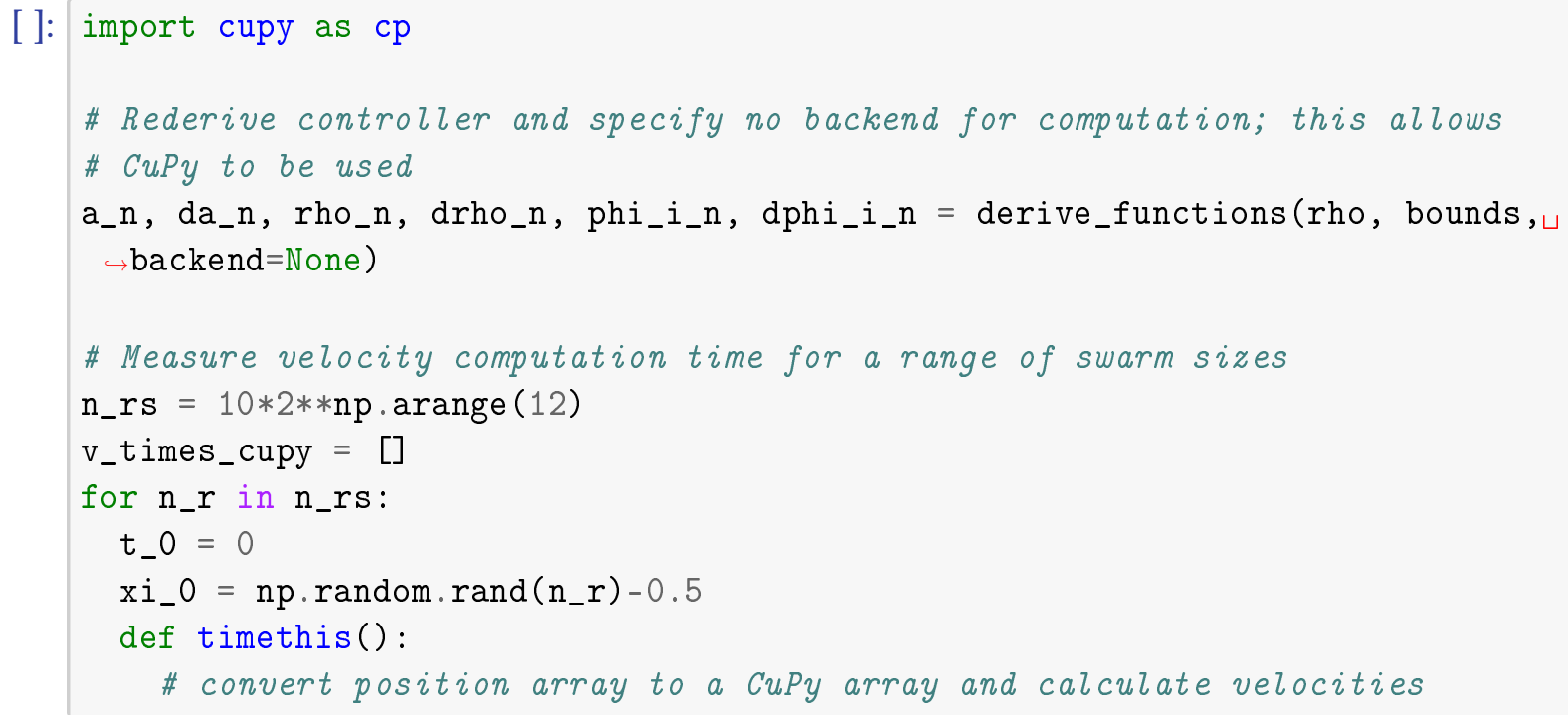



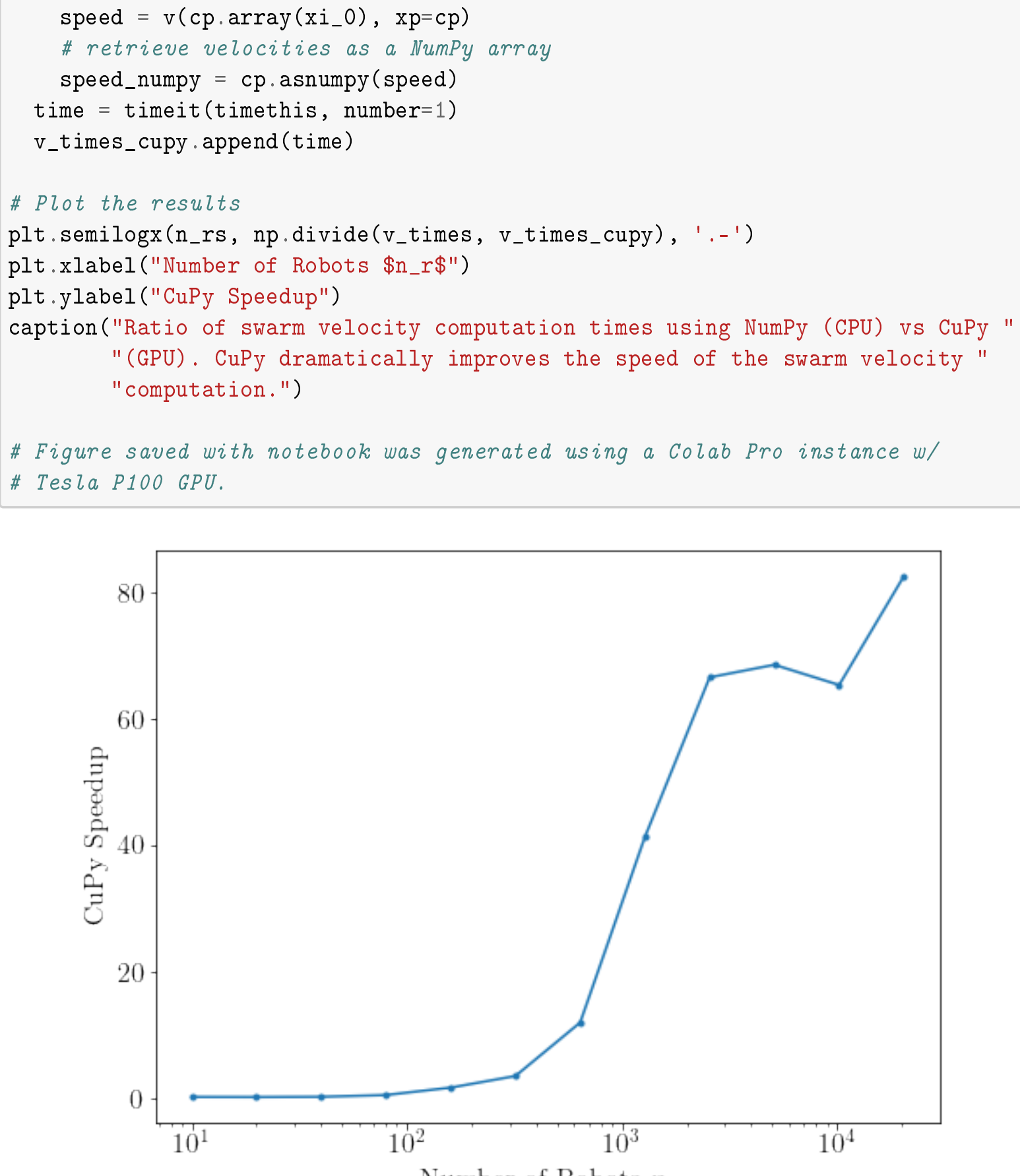

Number of Robots $n_{T}$

Figure 26. Ratio of swarm velocity computation times using NumPy (CPU) vs $\mathrm{CuPy}(\mathrm{GPU})$. CuPy dramatically improves the speed of the swarm velocity computation.

The speedup levels off as all the cores of the GPU become engaged, but even a constant speedup this large is sufficient to substantially increase the size of swarm that can be simulated in reasonable time. 
Unfortunately, the speedup in velocity calculation is not immediately reflected in the integration times.

[]: \# Time simulation using CuPy backend for a range of swarm sizes

exec_times_cupy $=[]$

$t=n p$. inspace $(0,2,100)$

def $\operatorname{dydt}(t, y)$ :

return $c p \cdot \operatorname{asnumpy}(v(c p \cdot \operatorname{array}(y)))$

n_rs $=$ n_rs $=(10 * 2 * * n p$.arange $(0,5.5,0.5))$.astype (int)

for $n_{-} r$ in $n_{-} r s$ :

$\mathrm{xi} 0=\mathrm{np} \cdot \mathrm{random} \cdot \mathrm{rand}\left(\mathrm{n}_{-} \mathrm{r}\right)-0.5$

def timethis():

sol $=$ solve_ivp (dydt, $(t[0], t[-1]), x i \_0$, events=event, method='BDF' $)$

time = timeit (timethis, number $=1$ )

exec_times_cupy.append(time)

\# Compare against simulation with NumPy backend

plt. $\log \log \left(n_{-} r s\right.$, exec_times, '.-', n_rs, exec_times_cupy, '.-')

plt.xlabel ("Number of Robots $\$ n_{-} r \$ "$ )

plt.ylabel("Simulation Execution Time \$T\$")

plt.legend(("Simulation Execution Time (NumPy)", "Simulation Execution Time $\rightarrow($ CuPy $) "))$

caption("Simulation execution times using NumPy (CPU) vs CuPy "

"(GPU). The maximum speedup (for $\$ n_{-} r=\{0\} \$$ robots) is $\$\{1: .2 f\} \backslash \backslash$ times $\$$."

"".format(n_rs[-1], exec_times[-1]/exec_times_cupy [-1]))

\# Figure saved with notebook was generated using a Colab Pro instance w/

\# Tesla P100 GPU. 


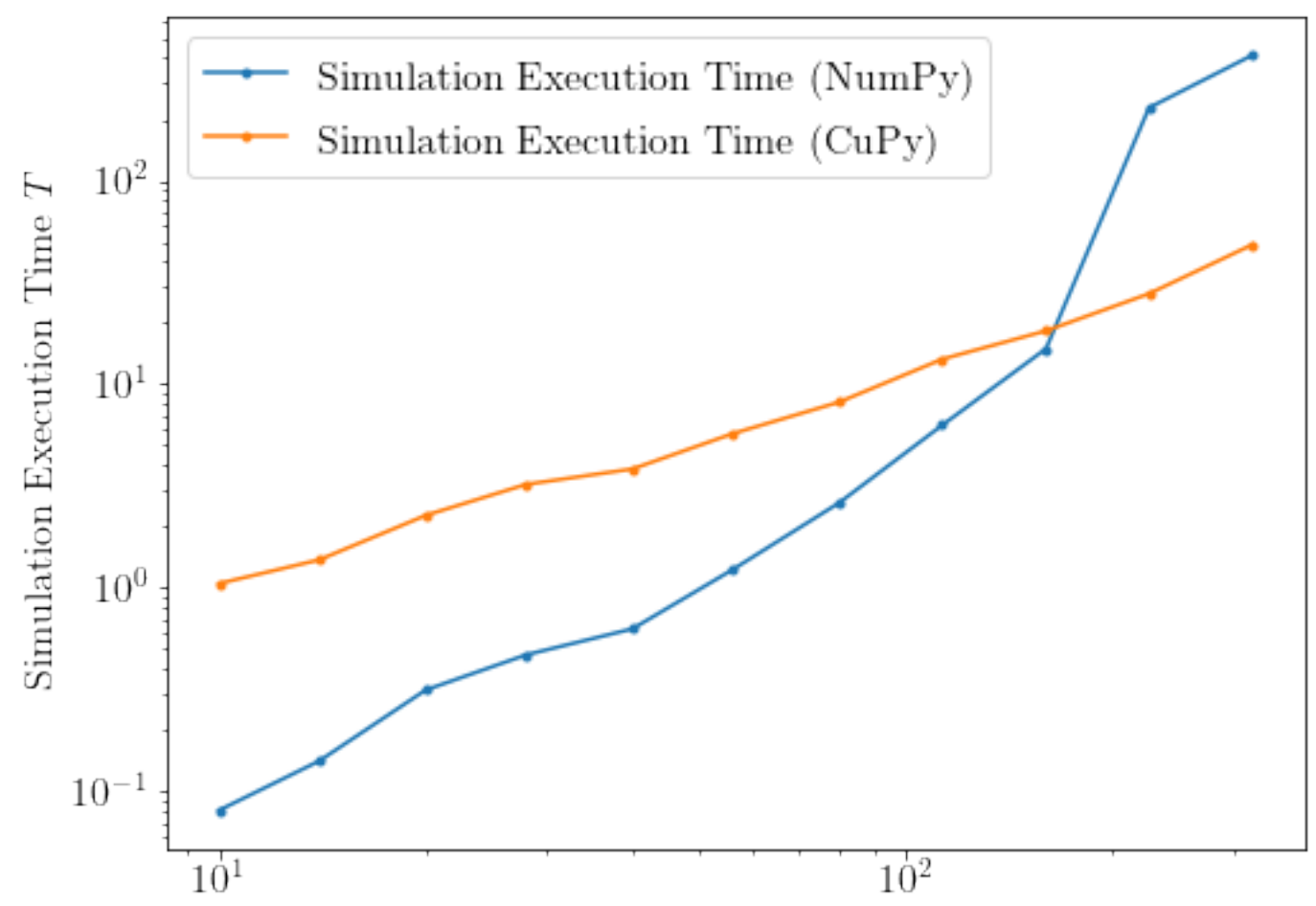

Number of Robots $n_{T}$

Figure 27. Simulation execution times using NumPy (CPU) vs CuPy (GPU). The maximum speedup (for $n_{r}=320$ robots) is $8.53 \times$.

While the GPU certainly improves performance for large swarms, the speedup (up to $8.5 \times$ ) is not quite as impressive as we would hope based on Figure 26. The reason is that every time dydt is called, the array containing the positions of the robots must by converted to a CuPy array and sent to the GPU. Upon completion, the evaluated velocities have to be returned to the CPU as a NumPy array in order for the integrator solve_ivp to solve for robot positions at the next time step. The overhead of this data conversion and transmission dramatically slows the integration. (We haven't found an integration suite in the SciPy ecosystem that keeps most or all computation on the GPU, so we are considering writing our own.)

In the meantime, we can substantially reduce the number of calls to dydt, and thus the overhead of communication with the GPU, using the vectorized option of solve_ivp. As mentioned above, each time the Jacobian is needed, solve_ivp calls dydt $n$ times, each time with a different $1 \mathrm{D}$ array of $n$ robot positions. By activating the vectorized option, solve_ivp will call dydt only once with a $2 \mathrm{D}$ array containing $n$ columns of $n$ robot positions.

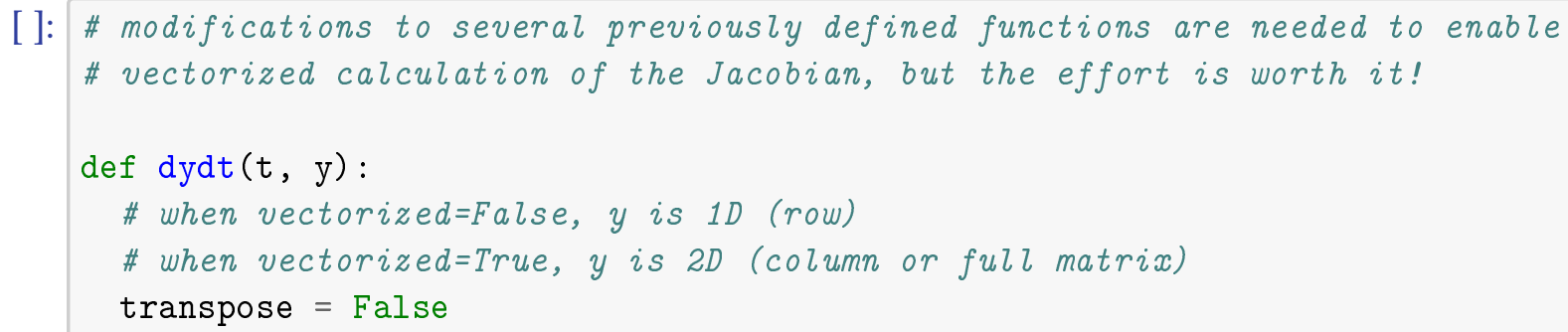




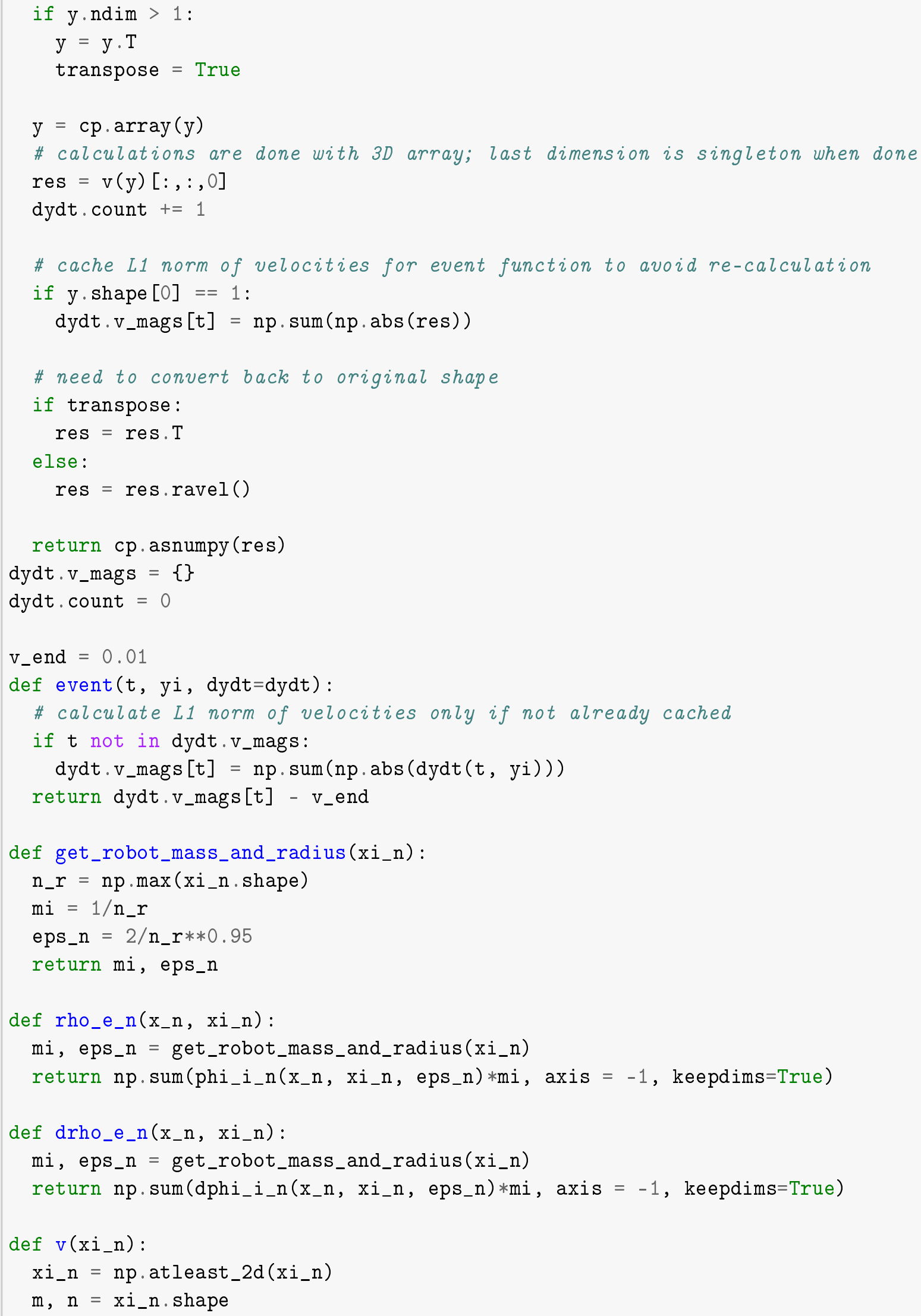




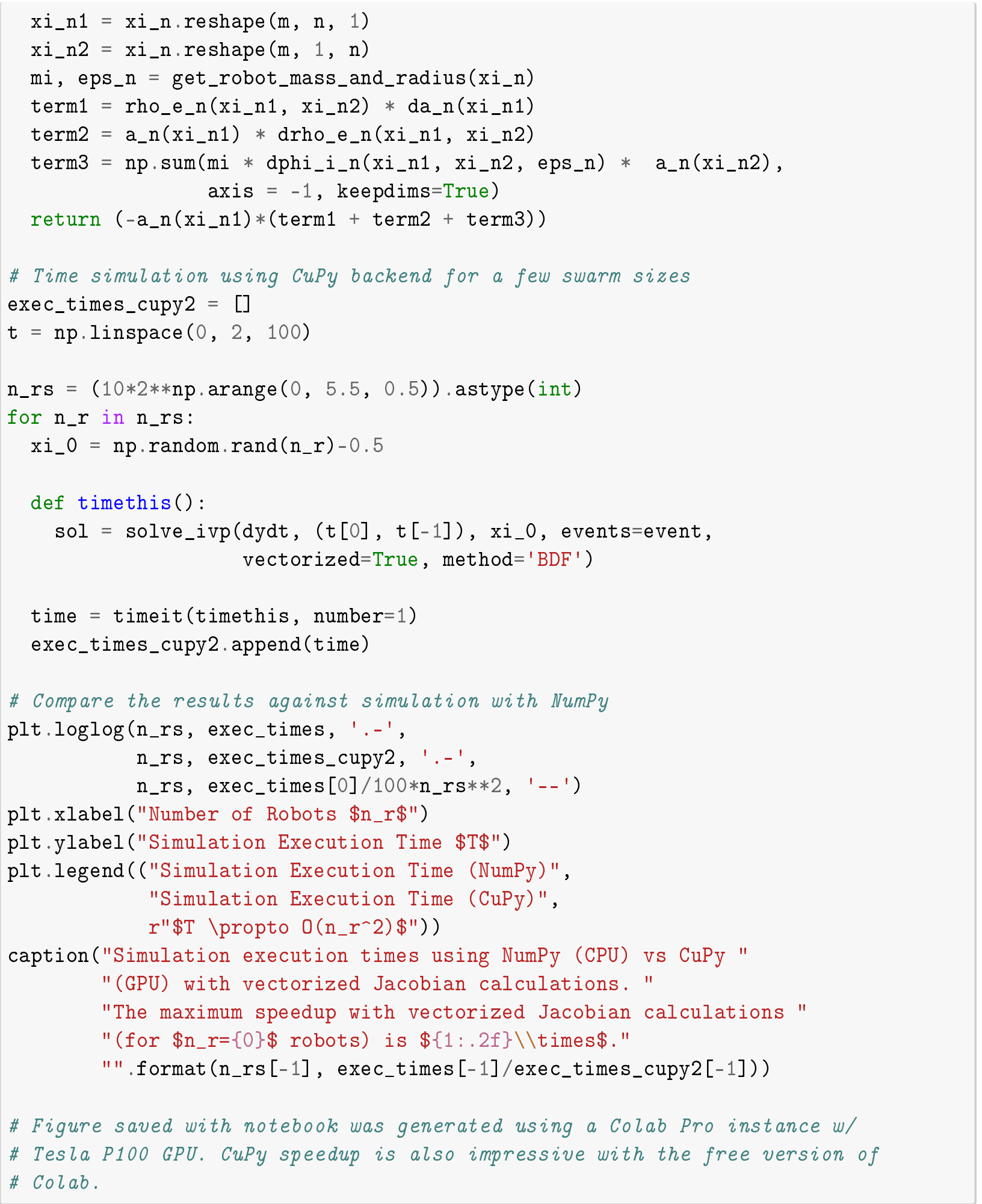




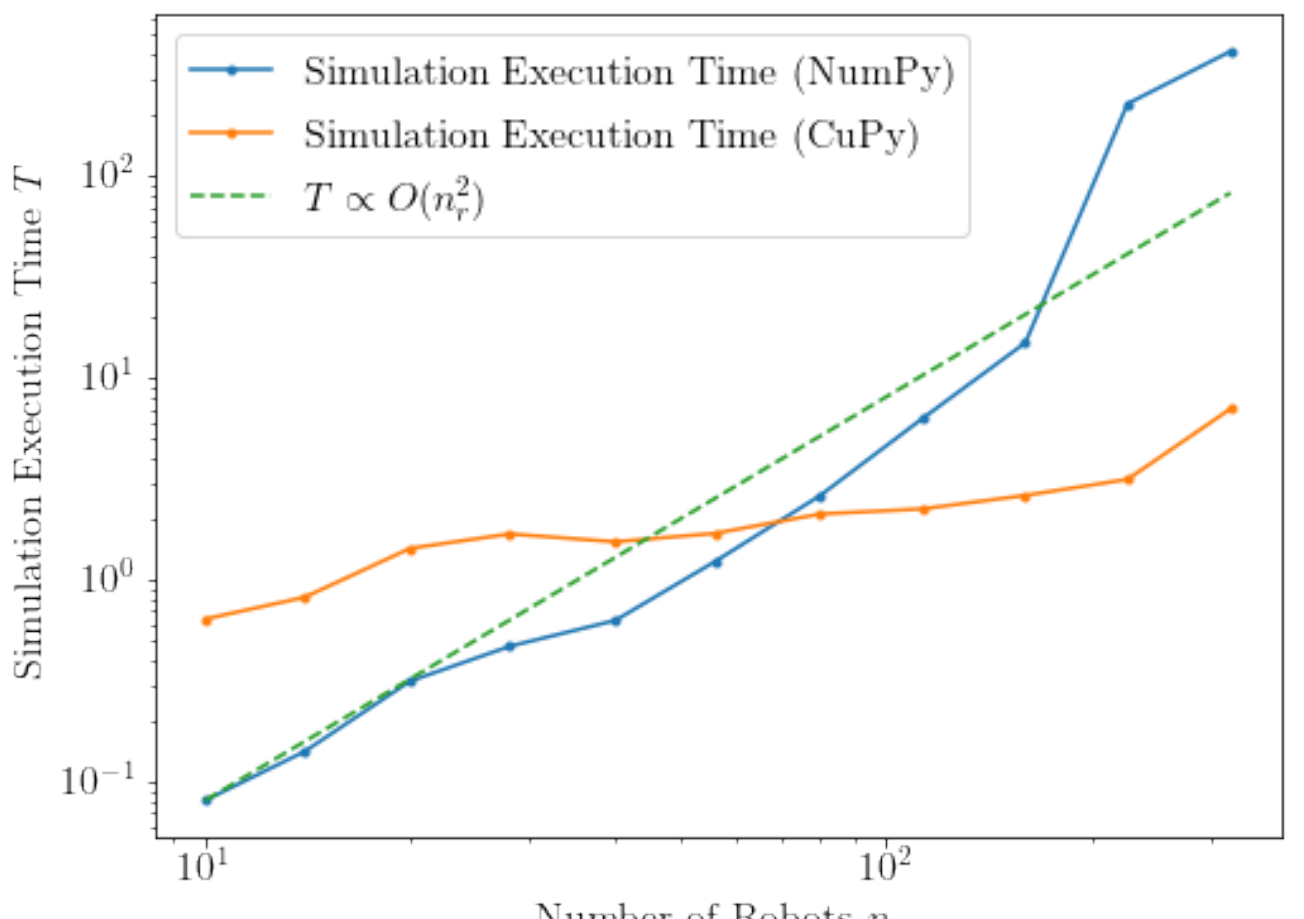

Figure 28. Simulation execution times using NumPy (CPU) vs CuPy (GPU) with vectorized Jacobian calculations. The maximum speedup with vectorized Jacobian calculations (for $n_{T}=320$ robots) is $59.01 \times$.

Use of a GPU with CuPy enables simulation of much larger swarms than was previously feasible.

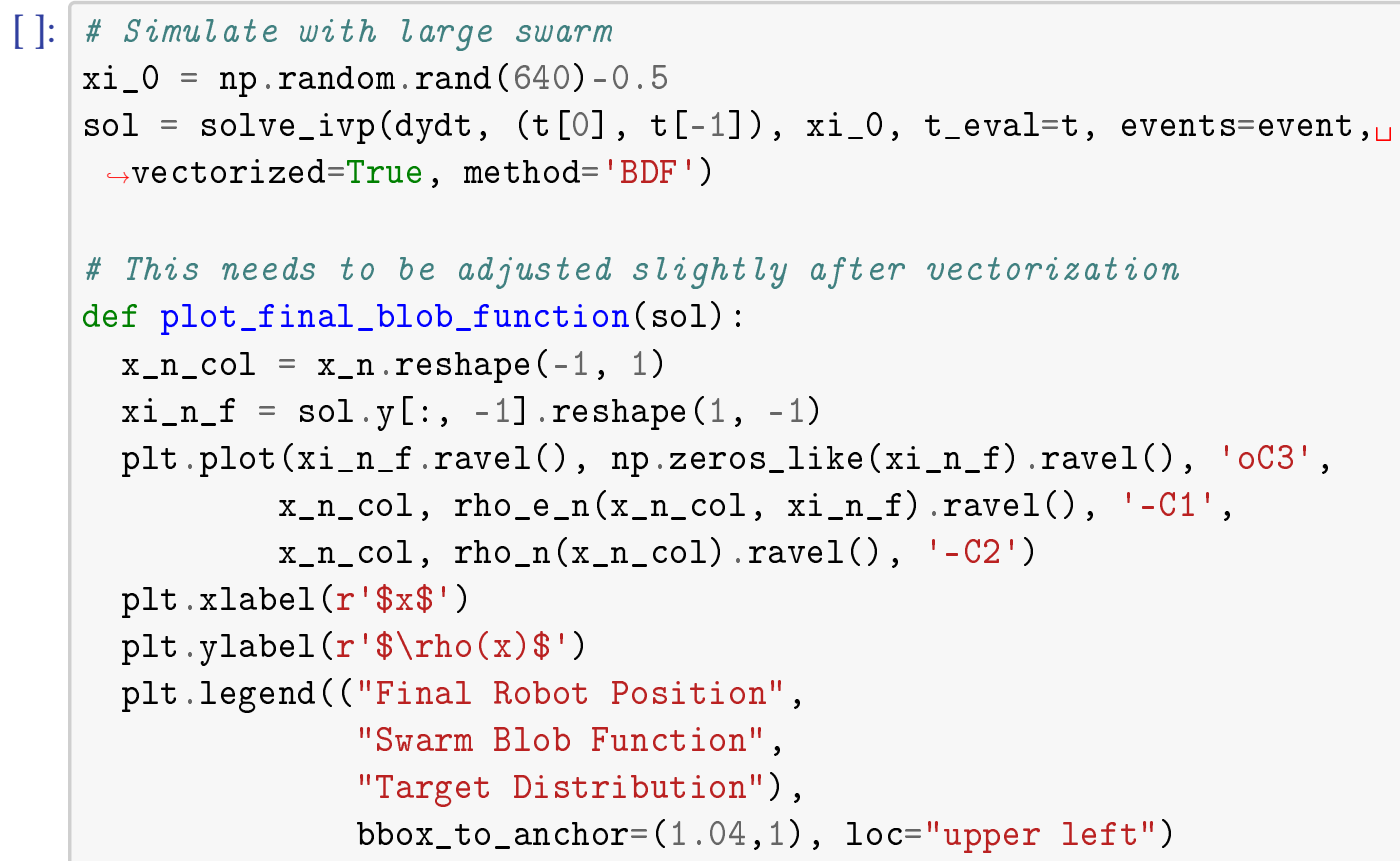



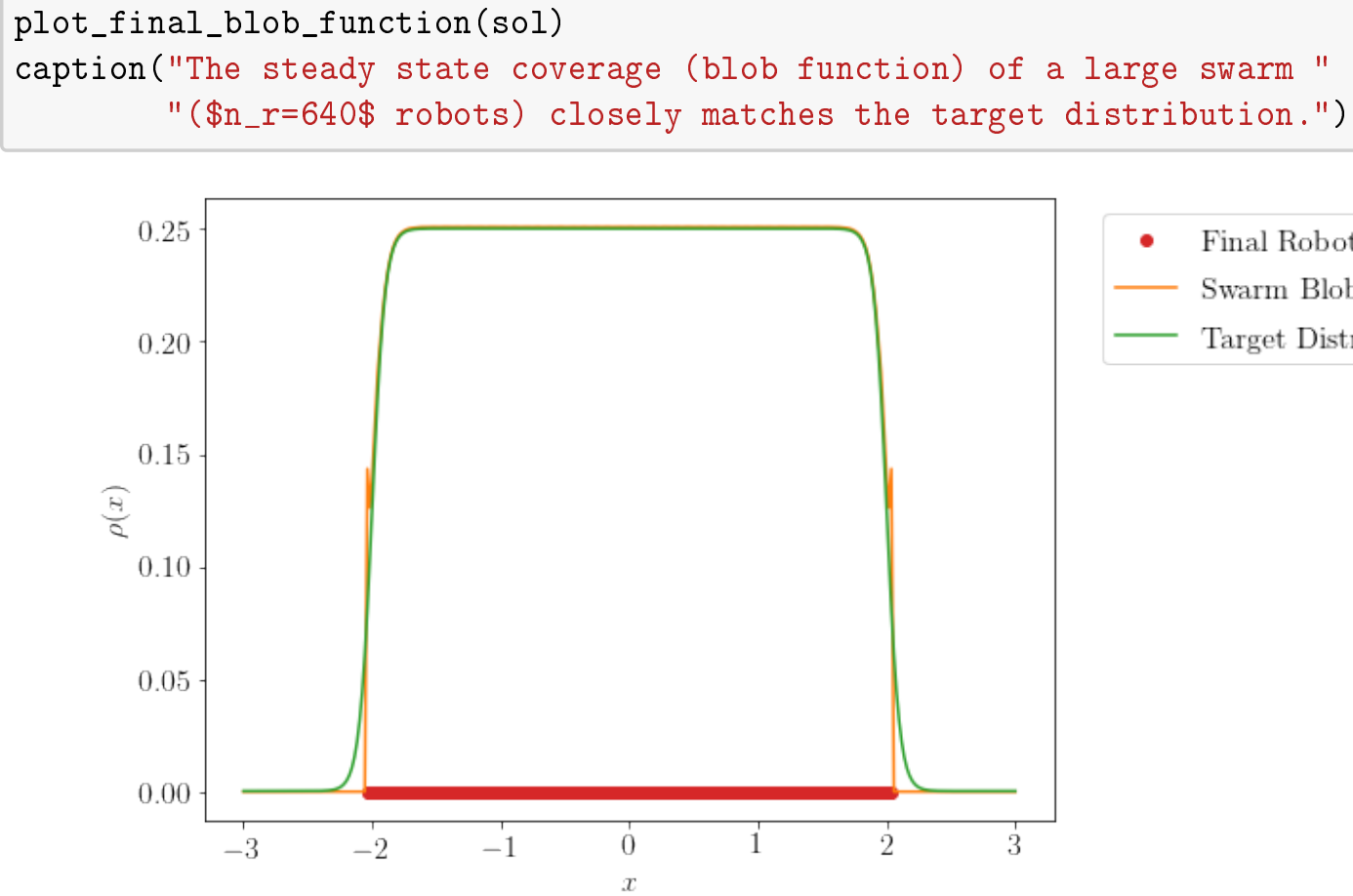

- Final Robot Position

- Swarm Blob Function

Target Distribution

Figure 29. The steady state coverage (blob function) of a large swarm $\left(n_{r}=640\right.$ robots) closely matches the target distribution.

\subsubsection{JIT Compilation}

There are a few other performance improvement ideas that we have not yet implemented. All stem from the fact that within (3), the quantity $e^{\frac{-\left(x_{i}-x_{j}\right)^{2}}{2 \epsilon^{2}}}$ appears several times. First, in the course of evaluating (3), this term is evaluated repeatedly with identical inputs; this redundancy should be eliminated. Second, we can exploit the symmetry of the pairwise interactions between robots, $e^{\frac{-\left(x_{i}-x_{j}\right)^{2}}{2 \epsilon^{2}}}=e^{\frac{-\left(x_{j}-x_{i}\right)^{2}}{2 \epsilon^{2}}}$, to reduce the number of calculations by a factor of approximately two. Finally, and most importantly, sufficiently distant robots have negligible effect on one another: $e^{\frac{-\left(x_{i}-x_{j}\right)^{2}}{2 \epsilon^{2}}} \approx 0$ when $\left(x_{i}-x_{j}\right)^{2} \gg \epsilon^{2}$. By partitioning the space, we can in $O(1)$ time determine which $O(1)$ interactions must be computed, and therefore we can exploit this sparsity of interactions to reduce the computational complexity of evaluating (3) to $O(n)$. These ideas do not seem easy to implement in a vectorized way, but use of a Python compiler such as Numba or Pythan could make them feasible.

\section{References}

[1] Carrillo, José Antonio, Katy Craig, and Francesco S. Patacchini. "A blob method for diffusion." Calculus of Variations and Partial Differential Equations 58.2 (2019): 53.

[2] Anderson, Brendon G., et al. "Quantifying Robotic Swarm Coverage." International Conference on Informatics in Control, Automation and Robotics. Springer, Cham, 2018. 